\title{
Row and Column Removal Theorems for Homomorphisms of Specht Modules and Weyl Modules
}

SINÉAD LYLE

ANDREW MATHAS

School of Mathematics and Statistics F07, University of Sydney, NSW 2006, Australia s.lyle@maths.usyd.edu.au a.mathas@maths.usyd.edu.au

\begin{abstract}
We prove a $q$-analogue of the row and column removal theorems for homomorphisms between Specht modules proved by Fayers and the first author [16]. These results can be considered as complements to James and Donkin's row and column removal theorems for decomposition numbers of the symmetric and general linear groups. In this paper we consider homomorphisms between the Specht modules of the Hecke algebras of type $A$ and between the Weyl modules of the $q$-Schur algebra.
\end{abstract}

Keywords: Hecke algebras, Schur algebras

\section{Introduction}

In his classic book on the representation theory of the symmetric groups James [22] conjectured that the decomposition numbers of the symmetric group should be invariant under a certain "column removal" operation. In a series of papers, James [23-25] and Donkin [9,13] proved a more general version of this result, together with a "row removal" analogue, not only for the symmetric groups, but also for the Schur algebras, the Iwahori-Hecke algebras of type $A$ and, finally, for the $q$-Schur algebras.

Quite recently, Fayers and the first author [16] showed that homomorphisms between Specht modules also satisfy these row and column removal theorems. In this paper we prove $q$-analogues of the row and column removal theorems for homomorphisms between Specht modules of the Hecke algebras of type $A$ and homomorphisms between Weyl modules of the $q$-Schur algebra. We also discuss recent generalizations of these results to Ext-groups and to "crystallized" decomposition numbers.

In order to state these results precisely, fix a positive integer $n \geq 1$ and let $\mathfrak{S}_{n}$ be the symmetric group of degree $n$. We also fix an integral domain $R$ and an invertible element $q$ in $R$. We define $e>1$ to be minimal such that $1+q+\cdots+q^{e-1}=0$. Let $\mathscr{H}_{n}=\mathscr{H}_{R, q}\left(\mathfrak{S}_{n}\right)$ be the Hecke algebra of $\mathfrak{S}_{n}$; that is, $\mathscr{H}_{n}$ is the unital associative $R$-algebra with generators

This research was supported by ARC grant DP0343023. The first author was also supported by a Sesqui Research Fellowship at the University of Sydney. 
$T_{1}, T_{2}, \ldots, T_{n-1}$ and relations

$$
\begin{aligned}
\left(T_{i}-q\right)\left(T_{i}+1\right) & =0, & & \text { for } i=1,2, \ldots, n-1, \\
T_{i} T_{j} & =T_{j} T_{i}, & & \text { for } 1 \leq i<j-1 \leq n-2, \\
T_{i+1} T_{i} T_{i+1} & =T_{i} T_{i+1} T_{i}, & & \text { for } i=1,2, \ldots, n-2 .
\end{aligned}
$$

Set $s_{i}$ to be the transposition $(i, i+1) \in \mathfrak{S}_{n}$. Then $\left\{s_{1}, \ldots, s_{n-1}\right\}$ generate $\mathfrak{S}_{n}$ so that if $w \in \mathfrak{S}_{n}$ then $w=s_{i_{1}} s_{i_{2}} \ldots s_{i_{k}}$ for some $i_{1}, \ldots, i_{k}$. If $k$ is minimal, we set $T_{w}=T_{i_{1}} \ldots T_{i_{k}}$. For $k$ minimal, $T_{w}$ is independent of the choice of expression $s_{i_{1}} \ldots s_{i_{k}}$ for $w$ and we say that $w$ has length $k=\ell(w)$. In fact, $\mathscr{H}_{n}$ is free as an $R$-module with basis $\left\{T_{w} \mid w \in \mathfrak{S}_{n}\right\}$; see, for example, [33, Theorem 1.13].

Recall that a partition of $n$ is a weakly decreasing sequence $\lambda=\left(\lambda_{1} \geq \lambda_{2} \geq \ldots\right)$ of non-negative numbers which sum to $n$. We write $|\lambda|=n$. For each partition $\lambda$ of $n$ there is a right $\mathscr{H}_{n}$-module $S(\lambda)$, called a Specht module; we will recall its construction in more detail in the next section. The Specht modules are important because they give a complete set of pairwise non-isomorphic irreducible $\mathscr{H}_{n}$-modules when $\mathscr{H}_{n}$ is semisimple. If $\mathscr{H}_{n}$ is not semisimple then the irreducible $\mathscr{H}_{n}$-modules are precisely the heads of the Specht modules $S(\lambda)$, where $\lambda$ runs over the $e$-restricted partitions of $n$. (A partition is $e$-restricted if $\lambda_{i}-\lambda_{i+1}<e$, for $i \geq 1$.) See [13,33] for details.

We need one more piece of notation before we can state the row removal homomorphism theorem for Specht modules. For the rest of this paper we also fix an integer $s$ with $1 \leq s<n$. For each partition $\lambda$ we define the following partitions:

$$
\lambda^{t}=\left(\lambda_{1}, \ldots, \lambda_{s}\right) \quad \lambda^{b}=\left(\lambda_{s+1}, \lambda_{s+2}, \ldots\right) .
$$

The superscripts stand for "top" and "bottom" respectively. Notice that rather than removing a single row, we are really cutting the partition into two pieces; Donkin calls this operation a horizontal $s$-cut.

Pictorially, the partitions $\lambda^{t}$ and $\lambda^{b}$ may be viewed as follows:
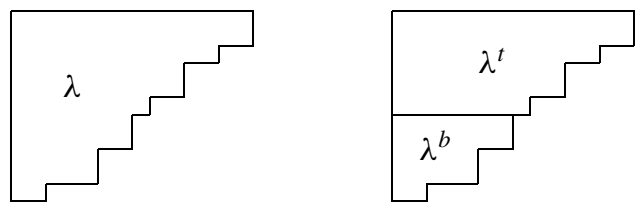

Theorem 1.1 Let $\lambda$ and $\mu$ be partitions of $n$ and assume that $q \neq-1$ or that $\mu^{t}$ is 2-restricted. Suppose that $\lambda_{1}+\cdots+\lambda_{s}=\mu_{1}+\cdots+\mu_{s}$. Then

$$
\operatorname{Hom}_{\mathscr{H}_{n}}(S(\mu), S(\lambda)) \cong \operatorname{Hom}_{\mathscr{H}_{n-m}}\left(S\left(\mu^{t}\right), S\left(\lambda^{t}\right)\right) \otimes \operatorname{Hom}_{\mathscr{H}_{m}}\left(S\left(\mu^{b}\right), S\left(\lambda^{b}\right)\right)
$$

where $m=\left|\lambda^{b}\right|$.

As with most of the results in this introduction, there is also an equivalent result describing homomorphisms under vertical $s$-cuts, or column removal; see Corollary 3.2 for details. For brevity we will consider only the "row removal" theorems in this introduction. 
In order to state the corresponding result for the $q$-Schur algebras recall that a composition of $n$ is a sequence $\mu=\left(\mu_{1}, \mu_{2}, \ldots\right)$ of non-negative numbers which sum to $n$. For each composition $\mu=\left(\mu_{1}, \ldots, \mu_{k}\right)$ of $n$ we have a Young subgroup $\mathfrak{S}_{\mu}=\mathfrak{S}_{\mu_{1}} \times \cdots \times \mathfrak{S}_{\mu_{k}}$ of $\mathfrak{S}_{n}$. Define $M(\mu)$ to be the right $\mathscr{H}_{n}$-module $M(\mu)=m_{\mu} \mathscr{H}_{n}$, where $m_{\mu}=\sum_{w \in \mathfrak{S}_{\mu}} T_{w}$.

Fix a non-negative integer $d$ and let $\Lambda(d, n)$ be the set of compositions of $n$ with at most $d$ non-zero parts. Let $\Lambda^{+}(d, n)$ be the set of partitions contained in $\Lambda(d, n)$. Dipper and James [7] defined the $q$-Schur algebra to be the endomorphism algebra

$$
\mathcal{S}_{q}(d, n)=\operatorname{End}_{\mathscr{H}_{\mathrm{n}}}\left(\bigoplus_{\mu \in \Lambda(d, n)} M(\mu)\right) .
$$

For each partition $\lambda \in \Lambda^{+}(d, n)$ there is a right $\mathcal{S}_{q}(d, n)$-module $\Delta(\lambda)$, called a Weyl module, or standard module. The head $L(\lambda)$ of $\Delta(\lambda)$ is irreducible and $\left\{L(\lambda) \mid \lambda \in \Lambda^{+}(d, n)\right\}$ is a complete set of pairwise non-isomorphic irreducible $\mathcal{S}_{q}(d, n)$-modules. See, for example, $[8,13,33]$.

We will deduce Theorem 1.1 from the following theorem for Weyl modules.

Theorem 1.2 Assume that $\lambda, \mu \in \Lambda^{+}(d, n)$. Suppose that $\lambda_{1}+\cdots+\lambda_{s}=\mu_{1}+\cdots+\mu_{s}$. Then

$$
\begin{aligned}
& \operatorname{Hom}_{\mathcal{S}_{q}(d, n)}(\Delta(\mu), \Delta(\lambda)) \\
& \quad \cong \operatorname{Hom}_{\mathcal{S}_{q}\left(d^{\prime}, n-m\right)}\left(\Delta\left(\mu^{t}\right), \Delta\left(\lambda^{t}\right)\right) \otimes \operatorname{Hom}_{\mathcal{S}_{q}\left(d^{\prime \prime}, m\right)}\left(\Delta\left(\mu^{b}\right), \Delta\left(\lambda^{b}\right)\right) .
\end{aligned}
$$

where $m=\left|\lambda^{b}\right|$ and $d^{\prime}, d^{\prime \prime}>0$ satisfy $\mu^{t}, \lambda^{t} \in \Lambda^{+}\left(d^{\prime}, n-m\right)$ and $\mu^{b}, \lambda^{b} \in \Lambda^{+}\left(d^{\prime \prime}, m\right)$.

We prove Theorems 1.1 and 1.2 by giving explicit combinatorially defined bijections between the two Hom-spaces involved.

For the moment consider the special case when $d=n$. Then the identity map $\varphi_{\omega}: \mathscr{H}_{n} \rightarrow$ $\mathscr{H}_{n}$ on $\mathscr{H}_{n}$ can be considered as an element of $\mathcal{S}_{q}(n, n)$. By a standard $\operatorname{argument}, \mathscr{H}_{n} \cong$ $\varphi_{\omega} \mathcal{S}_{q}(n, n) \varphi_{\omega}$. Hence, there is a functor, known as the Schur functor, from the category of right $\mathcal{S}_{q}(n, n)$-modules to the category of right $\mathscr{H}_{n}$-modules which is given by

$$
\alpha: \operatorname{Mod}-\mathcal{S}_{q}(n, n) \rightarrow \operatorname{Mod}-\mathscr{H}_{n} ; M \mapsto M \varphi_{\omega} .
$$

Furthermore, the Schur functor is exact because $\varphi_{\omega}$ is an idempotent, so $\varphi_{\omega} \mathcal{S}_{q}(d, n)$ is projective.

It is easy to see that the Schur functor maps Weyl modules to Specht modules. Consequently, one might expect that Theorem 1.1 follows from Theorem 1.2 simply by applying the Schur functor. In fact this is not the case. The problem is that the inverse Schur functor

$$
\beta: \operatorname{Mod}-\mathscr{H}_{n} \rightarrow \operatorname{Mod}-\mathcal{S}_{q}(n, n) ; N \mapsto N \otimes_{\mathscr{H}_{n}} \mathcal{S}_{q}(n, n)
$$

does not, in general, send Specht modules to Weyl modules. Consequently, the homomorphism $\operatorname{Hom}_{\mathcal{S}_{q}(n, n)}(\Delta(\mu), \Delta(\lambda)) \rightarrow \operatorname{Hom}_{\mathscr{H}_{n}}(S(\mu), S(\lambda))$ given by the Schur functor is 
not necessarily surjective. Nevertheless, generalizing a famous classical result of Carter and Lusztig [2], Dipper and James [8, Corollary 5.6] proved that if $q \neq-1$, or if $\mu$ is 2-restricted, then

$$
\operatorname{Hom}_{\mathcal{S}_{q}(n, n)}(\Delta(\mu), \Delta(\lambda)) \cong \operatorname{Hom}_{\mathscr{H}_{n}}(S(\mu), S(\lambda)) .
$$

Hence, Theorem 1.1 follows from Theorem 1.2. Dipper and James' proof of this result is quite long, so we give a short proof of (1.3) in the next section.

Theorems 1.1 and 1.2 have well-known counterparts for decomposition numbers. If $\Delta$ is a $\mathcal{S}_{q}(d, n)$-module and $L$ is an irreducible $\mathcal{S}_{q}(d, n)$-module, let $[\Delta: L]$ be the composition multiplicity of $L$ in $\Delta$.

Theorem 1.4 (James [25] and Donkin [13]) Suppose that $R$ is a field and that $\lambda, \mu \in$ $\Lambda^{+}(d, n)$. Suppose that $\lambda_{1}+\cdots+\lambda_{s}=\mu_{1}+\cdots+\mu_{s}$. Then

$$
[\Delta(\mu): L(\lambda)]=\left[\Delta\left(\mu^{t}\right): L\left(\lambda^{t}\right)\right] \times\left[\Delta\left(\mu^{b}\right): L\left(\lambda^{b}\right)\right] .
$$

where $m=\left|\lambda^{b}\right|$ and the two right hand decomposition multiplicities are computed in suitable $q$-Schur algebras $\mathcal{S}_{q}\left(d^{\prime}, n-m\right)$ and $\mathcal{S}_{q}\left(d^{\prime \prime}, m\right)$.

Unlike the results concerning homomorphisms, the Schur functor can be applied directly here to deduce the analogous results for the decomposition numbers of $\mathscr{H}_{n}$. Specifically, if $S$ is an $\mathscr{H}_{n}$-module and $D$ is a simple $\mathscr{H}_{n}$-module let $[S: D]$ be the decomposition multiplicity of $D$ in $S$. If $\lambda$ is an $e$-restricted partition, let $D(\lambda)=S(\lambda) / \operatorname{rad} S(\lambda)$ be the corresponding simple $\mathscr{H}_{n}$-module (see, for example, [33]). Then, under the assumptions of Theorem 1.1, if $\lambda, \lambda^{t}$ and $\lambda^{b}$ are $e$-restricted then

$$
[S(\mu): D(\lambda)]=\left[S\left(\mu^{t}\right): D\left(\lambda^{t}\right)\right] \times\left[S\left(\mu^{b}\right): D\left(\lambda^{b}\right)\right] .
$$

One way to prove Theorem 1.4 is to view the Weyl modules as modules for the quantum general linear group $G(d)$. As is well known [13, Section 0.21], $\operatorname{Mod}-\mathcal{S}_{q}(d, n)$ is the full subcategory of Mod- $G(d)$ consisting of the homogeneous polynomial representations of degree $n$. Let $\Pi$ be a set of simple roots for $G(d)$ and let $G_{\Sigma}(d)$ be a Levi quantum subgroup of $G(d)$ corresponding to a subset $\Sigma \subseteq \Pi$. Let $X$ be the set of weights for $G(d)$ and $X^{+}$be the set of dominant weights. Similarly, let $X_{\Sigma}$ and $X_{\Sigma}^{+}$be the weights and dominant weights for $G_{\Sigma}(d)$. Finally, write $\Delta_{\Sigma}(\mu)$, and $L_{\Sigma}(\mu)$ for the Weyl modules (or standard modules), and the simple modules, respectively, of $G_{\Sigma}(d)$; see, for example, [13, Section 4.2].

Recall that, as an $R$-module, every $\mathcal{S}_{q}(d, n)$-module $M$ decomposes into a direct sum of weight spaces: $M=\oplus_{\mu \in \Lambda(d, n)} M_{\mu}$, where $M_{\mu}=M \varphi_{\mu}$ and $\varphi_{\mu}: M(\mu) \rightarrow M(\mu)$ is the identity map. For each weight $\lambda \in X_{\Sigma}^{+}$, define the "truncation functor"

$$
\operatorname{Tr}_{\Sigma}^{\lambda}: \operatorname{Mod}-G(d) \rightarrow \operatorname{Mod}-G_{\Sigma}(d) ; M \mapsto \bigoplus_{\substack{\mu \in X \\ \lambda-\mu \in \mathbb{Z}}} M_{\mu} .
$$


These functors are implicit in Green's classic book [18] and James [23] essentially used a special case of them (corresponding to $s=1$ ) in his proof of Theorem 1.4 for the symmetric groups $(q=1)$. The functors were introduced by Donkin $[10,11]$ who used them to study not only representations of the Schur algebras but also a more general class of Schur algebras associated with arbitrary algebraic groups; see [26].

Donkin [13, Section 4.2(4)] shows that if $\mu \in X^{+}$and $\lambda-\mu \in \mathbb{Z} \Sigma$ then $\operatorname{Tr}_{\Sigma}^{\lambda} \Delta(\mu) \cong$ $\Delta_{\Sigma}(\mu)$ and $\operatorname{Tr}_{\Sigma}^{\lambda} L(\mu) \cong L_{\Sigma}(\mu)$. Write $\Pi=\left\{\alpha_{1}, \ldots, \alpha_{d-1}\right\}$ for the simple roots of $G(d)$ where, as usual, $\alpha_{i}=\epsilon_{i}-\epsilon_{i+1}$. Now let $\Sigma=\Pi \backslash\left\{\alpha_{s}\right\}$. Then $G_{\Sigma}(d) \cong G(s) \times G(d-s)$ and $\Delta_{\Sigma}(v) \cong \Delta\left(v^{t}\right) \otimes \Delta\left(v^{b}\right)$, for all $v \in X_{\Sigma}^{+}$. The condition that $\lambda_{1}+\cdots+\lambda_{s}=\mu_{1}+\cdots+\mu_{s}$ is equivalent to the requirement that $\lambda-\mu \in \mathbb{Z} \Sigma$. It follows that

$$
\begin{aligned}
{[\Delta(\mu): L(\lambda)] } & =\left[\operatorname{Tr}_{\Sigma}^{\lambda} \Delta(\mu): \operatorname{Tr}_{\Sigma}^{\lambda} L(\lambda)\right] \\
& =\left[\Delta_{\Sigma}(\mu): L_{\Sigma}(\lambda)\right] \\
& =\left[\Delta\left(\mu^{t}\right) \otimes \Delta\left(\mu^{b}\right): L\left(\lambda^{t}\right) \otimes L\left(\lambda^{b}\right)\right] \\
& =\left[\Delta\left(\mu^{t}\right): L\left(\lambda^{t}\right)\right] \times\left[\Delta\left(\mu^{b}\right): L\left(\lambda^{b}\right)\right] .
\end{aligned}
$$

We have just sketched a proof of Theorem 1.4.

In Section 3 we prove Theorem 1.2 by constructing an explicit isomorphism between the two Hom-spaces whenever $R$ is an integral domain. Our proof relies upon a combinatorial description of the Hom-spaces $\operatorname{Hom}_{\mathcal{S}_{q}(d, n)}\left(\varphi_{\mu} \mathcal{S}_{q}(d, n), \Delta(\lambda)\right)$. Observe that $\varphi_{\mu} \mathcal{S}_{q}(d, n)$ is projective, so the sequence $\varphi_{\mu} \mathcal{S}_{q}(d, n) \rightarrow \Delta(\mu)$ can be extended to a projective resolution of $\Delta(\mu)$. It might be possible to compute higher Ext-groups using our combinatorial methods if we had explicit projective resolutions of the Weyl modules; unfortunately, no such resolutions are known in general.

Using algebraic group techniques, however, Theorems 1.1 and 1.2 can be generalized to compute higher Ext-groups. To discuss these results we use the following (new) theorem of Donkin, which simultaneously generalizes Theorem 1.2, [5, Corollary 10] and [13, Section 4.2(17)]. With Donkin's permission, we include a proof of this result in Section 4.

We write $\lambda \geq \mu$ if $\lambda-\mu \in \mathbb{N}_{0} X^{+}$. When we identify weights and partitions, this ordering coincides with the usual dominance ordering on partitions.

Theorem 1.5 (Donkin) Suppose that $R$ is a field, $\Sigma \subset \Pi$ and let $\lambda$ and $\mu$ be dominant weights with $\lambda-\mu \in \mathbb{Z} \Sigma$. Assume that $V$ is a finite dimensional $G(d)$-module such that $V_{\nu} \neq 0$ only if $\lambda \geq v$. Then

$$
\operatorname{Ext}_{G(d)}^{k}(\Delta(\mu), V) \cong \operatorname{Ext}_{G_{\Sigma}(d)}^{k}\left(\Delta_{\Sigma}(\mu), \operatorname{Tr}_{\Sigma}^{\lambda} V\right),
$$

for all $k \geq 0$.

In particular, putting $V=\Delta(\lambda)$ in Theorem 1.5 shows that

$$
\begin{aligned}
\operatorname{Ext}_{G(d)}^{k}(\Delta(\mu), \Delta(\lambda)) & \cong \operatorname{Ext}_{G_{\Sigma}(d)}^{k}\left(\Delta_{\Sigma}(\mu), \Delta_{\Sigma}(\lambda)\right) \\
& =\operatorname{Ext}_{G(s) \times G(d-s)}^{k}\left(\Delta\left(\mu^{t}\right) \otimes \Delta\left(\mu^{b}\right), \Delta\left(\lambda^{t}\right) \otimes \Delta\left(\lambda^{b}\right)\right) .
\end{aligned}
$$


Now, if $U$ and $V$ are $\mathcal{S}_{q}(d, n)$-modules then $\operatorname{Ext}_{G(d)}^{k}(U, V) \cong \operatorname{Ext}_{\mathcal{S}_{q}(d, n)}^{k}(U, V)$, for $k \geq 0[12$, Section 4]. Hence, in the special case when $\Sigma=\Pi \backslash\left\{\alpha_{s}\right\}$, the Künneth formula now gives the following.

Corollary 1.6 (Donkin [13, Section 4.2(17)]) Assume that $R$ is a field, $\lambda, \mu \in \Lambda^{+}(d, n)$ and that $k \geq 0$. Suppose that $\lambda_{1}+\cdots+\lambda_{s}=\mu_{1}+\cdots+\mu_{s}$. Then

$$
\begin{aligned}
& \operatorname{Ext}_{\mathcal{S}_{q}(d, n)}^{k}(\Delta(\mu), \Delta(\lambda)) \\
& \cong \bigoplus_{i+j=k} \operatorname{Ext}_{\mathcal{S}_{q}\left(d^{\prime}, n-m\right)}^{i}\left(\Delta\left(\mu^{t}\right), \Delta\left(\lambda^{t}\right)\right) \otimes \operatorname{Ext}_{\mathcal{S}_{q}\left(d^{\prime \prime}, m\right)}^{j}\left(\Delta\left(\mu^{b}\right), \Delta\left(\lambda^{b}\right)\right),
\end{aligned}
$$

where $m=\left|\lambda^{b}\right|$ and $d^{\prime}, d^{\prime \prime}>0$ satisfy $\mu^{t}, \lambda^{t} \in \Lambda^{+}\left(d^{\prime}, n-m\right)$ and $\mu^{b}, \lambda^{b} \in \Lambda^{+}\left(d^{\prime \prime}, m\right)$.

Notice that if $k=0$ then Corollary 1.6 becomes Theorem 1.2, except that 1.2 allows $R$ to be an arbitrary integral domain. Notice that if $f \in \operatorname{Hom}_{\mathcal{S}_{q}(d, n)}(\Delta(\mu), \Delta(\lambda))$ then $\operatorname{Tr}_{\Sigma}^{\lambda} f$ is just the restriction of $f$ to $\operatorname{Tr}_{\Sigma}^{\lambda} \Delta(\mu) \cong \Delta\left(\mu^{t}\right) \otimes \Delta\left(\mu^{b}\right)$. So, in principle, when $k=0$ the functor $\operatorname{Tr}_{\Sigma}^{\lambda}$ induces a bijection $f \mapsto \operatorname{Tr}_{\Sigma}^{\lambda} f \mapsto f^{t} \otimes f^{b}$ between the two Hom-spaces in the Corollary. Our proof of Theorem 1.6 describes such a bijection explicitly in terms of semistandard homomorphisms. Using Proposition 2.10 it is possible to show that these two bijections coincide.

Once again, we cannot simply apply the Schur functor to Corollary 1.6 to produce an analogous result for the Ext-groups of the Specht modules. The inverse Schur functor $\beta$ is only right exact; by studying its left derived functors Donkin [14, Proposition 10.2] shows that

$$
\operatorname{Ext}_{\mathscr{H}_{n}}^{k}(\alpha \Delta(\mu), S(\lambda)) \cong \operatorname{Ext}_{\mathcal{S}_{q}(n, n)}^{k}(\Delta(\mu), \beta S(\lambda)), \quad \text { whenever } 0 \leq k<e-2 .
$$

(Note that Donkin uses the Dipper-James Specht modules [6] which are dual to ours.) The main point is that these Ext-groups can be computed using a Grothendieck spectral sequence. (This sequence collapses because if $I$ is an injective $\mathcal{S}_{q}(n, n)$-module then $\alpha I$ is a summand of $M(v)$, for some $v$, and $\operatorname{Ext}_{\mathscr{H}_{n}}^{k}(M(\nu), S(\lambda))=0$ by [14, Proposition 10.1].)

Hemmer and Nakano [21] have shown that Specht filtration multiplicities are uniquely determined for the symmetric groups in characteristic $p>3$. Donkin establishes a $q$ analogue of this result and uses it to prove that $\beta$ maps Specht modules to Weyl modules whenever $e>3$. These results, together with Corollary 1.6, imply the following.

Corollary 1.8 (Donkin [14, Proposition 10.4]) Assume that $R$ is a field, $q \neq-1, \lambda, \mu \in$ $\Lambda^{+}(d, n)$ and that $0 \leq k<e-2$. Suppose that $\lambda_{1}+\cdots+\lambda_{s}=\mu_{1}+\cdots+\mu_{s}$. Then

$$
\operatorname{Ext}_{\mathscr{H}_{n}}^{k}(S(\mu), S(\lambda)) \cong \bigoplus_{i+j=k} \operatorname{Ext}_{\mathscr{H}_{n-m}^{i}}\left(S\left(\mu^{t}\right), S\left(\lambda^{t}\right)\right) \otimes \operatorname{Ext}_{\mathscr{H}_{m}^{j}}^{j}\left(S\left(\mu^{b}\right), S\left(\lambda^{b}\right)\right),
$$

where $m=\left|\lambda^{b}\right|$.

Notice that the last step in Donkin's proof of Corollary 1.8, as sketched above, required $e>3$. However, if $e=3$ then the restrictions on $k$ force $k=0$. So Corollary 1.8 becomes 
a special case of Theorem 1.1 when $e=3$. We do not know how sharp the restrictions on $k$ are in Corollary 1.8; they are not exact, however, because if $e=2$ then Corollary 1.8 also holds when $k=0$. The restrictions on $k$ come from (1.7); some restriction is needed here because $\mathcal{S}_{q}(d, n)$ has finite global dimension (since $\mathcal{S}_{q}(d, n)$ is quasi-hereditary) whereas $\mathscr{H}_{n}$ does not $\left(\mathscr{H}_{n}\right.$ is self-injective).

We next want to relate these Ext-group calculations to decomposition numbers. Taking $V=L(\lambda)$ in Theorem 1.5 we obtain the following.

Corollary 1.9 (Cline, Parshall and Scott [5, Corollary 10]) Suppose that $R$ is a field, $\Sigma \subset \Pi$ and let $\lambda$ and $\mu$ be dominant weights with $\lambda-\mu \in \mathbb{Z} \Sigma$. Then

$$
\operatorname{Ext}_{G(d)}^{k}(\Delta(\mu), L(\lambda)) \cong \operatorname{Ext}_{G_{\Sigma}(d)}^{k}\left(\Delta_{\Sigma}(\mu), L_{\Sigma}(\lambda)\right),
$$

for all $k \geq 0$.

Once again, suppose that $\Sigma=\Pi \backslash\left\{\alpha_{s}\right\}$ and that $\lambda_{1}+\cdots+\lambda_{s}=\mu_{1}+\cdots+\mu_{s}$. Applying the Künneth formula to the right hand side of the last equation we find

$$
\operatorname{Ext}_{\mathcal{S}_{q}(d, n)}^{k}(\Delta(\mu), L(\lambda)) \cong \bigoplus_{i+j=k} \operatorname{Ext}_{\mathcal{S}_{q}\left(d^{\prime}, n-m\right)}^{i}\left(\Delta\left(\mu^{t}\right), L\left(\lambda^{t}\right)\right) \otimes \operatorname{Ext}_{\mathcal{S}_{q}\left(d^{\prime \prime}, m\right)}^{j}\left(\Delta\left(\mu^{b}\right), L\left(\lambda^{b}\right)\right),
$$

where $m, d^{\prime}$ and $d^{\prime \prime}$ are as above. For the cases $s=1$ and $s=\max \left\{l \mid \lambda_{l}>0\right\}$, see [5, Theorem 13].

Given an $\mathcal{S}_{q}(d, n)$-module $M$ let $\operatorname{ch} M=\sum_{\mu}\left(\operatorname{dim} M \varphi_{\mu}\right) e^{\mu}$ be its (formal) character. If $\mu$ is a dominant weight then ch $\Delta(\mu)$ is given explicitly by the Weyl character formula. By definition, we also have ch $\Delta(\mu)=\sum_{\lambda}[\Delta(\mu): L(\lambda)] \operatorname{ch} L(\lambda)$. In contrast, by Delorme's theorem [26, Section II.6.21(6)], we know that

$$
\operatorname{ch} L(\lambda)=\sum_{\mu}\left\{\sum_{k \geq 0}(-1)^{k} \operatorname{dim} \operatorname{Ext}_{\mathcal{S}_{q}(d, n)}^{k}(\Delta(\mu), L(\lambda))\right\} \operatorname{ch} \Delta(\mu) .
$$

Thus, the spaces $\operatorname{Ext}_{\mathcal{S}_{q}(d, n)}^{k}(\Delta(\mu), L(\lambda))$ determine the (inverse) decomposition matrix of $\mathcal{S}_{q}(d, n)$.

In order to exploit (1.11) we follow [4] and define polynomials $Q_{\mu \lambda}(t) \in \mathbb{N}_{0}[t]$ by

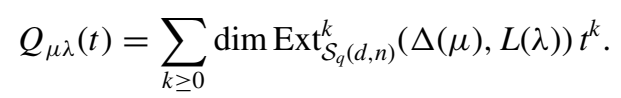

It follows from Kempf's vanishing theorem that: (1) this sum is finite; (2) $Q_{\lambda \lambda}(t)=1$; (3) if $\mu \neq \lambda$ then $Q_{\mu \lambda} \in t \mathbb{N}_{0}[t]$; and (4) $Q_{\mu \lambda} \neq 0$ only if $\lambda \geq \mu$. (See [26, Section II.4.13 Remark 2], for the classical case; using the $q$-analogue of Kempf's vanishing theorem the same argument works in general.) Note also that $\operatorname{ch} L(\lambda)=\sum_{\mu} Q_{\mu \lambda}(-1) \operatorname{ch} \Delta(\mu)$ by (1.11). 
We want to utilize the polynomials $Q_{\mu \lambda}(t)$ in the special case when $R=\mathbb{C}$ and $q$ is an $e^{\text {th }}$ root of unity. In this case, work of Varagnolo and Vasserot [35] and Leclerc and Thibon [32] gives an algorithm for computing the decomposition numbers of $\mathcal{S}_{q}(d, n)$. This algorithm amounts to computing a certain basis $\left\{B_{\lambda}\right\}$ of the combinatorial Fock space $\mathcal{F}$ which, as a $\mathbb{Q}\left[t, t^{-1}\right]$-module, is free with a basis given by the set of all partitions of all non-negative integers. If $\lambda \in \Lambda^{+}(d, n)$ then the basis element $B_{\lambda}$ can be characterized as the unique element of $\mathcal{F}$ which is (1) invariant under a certain "bar involution", and (2) of the form

$$
B_{\lambda}=\sum_{\mu \in \Lambda^{+}(d, n)} d_{\mu \lambda}(t) \mu,
$$

where $d_{\lambda \lambda}=1$ and $d_{\mu \lambda}(t) \in t^{2} \mathbb{Z}\left[t^{2}\right]$ if $\lambda \neq \mu$. In fact, $B_{\lambda}$ is an element of the "canonical basis" of $\mathcal{F}$ when it is considered as a module for an affine quantum group; see [32,35].

The Lusztig conjecture for quantum groups at a complex root of unity was established by Kazhdan and Lusztig [28] and Kashiwara and Tanisaki [27]. Building on this work, Varagnolo and Vasserot [35] proved that the polynomials $d_{\mu \lambda}(t)$ are parabolic KazhdanLusztig polynomials for the affine Weyl group $\widehat{\mathfrak{S}_{d}}$ and, moreover, that $d_{\mu \lambda}(1)=[\Delta(\mu)$ : $L(\lambda)]$.

Let $\mathbf{D}_{d, n}(t)=\left(d_{\lambda \mu}(t)\right)_{\lambda, \mu \in \Lambda^{+}(d, n)}$. Then $\mathbf{D}_{d, n}(1)$ is the decomposition matrix of $\mathcal{S}_{q}(d, n)$ by Varagnolo and Vasserot's theorem. Also, by the remarks above, $\mathbf{D}_{d, n}(t)$ is a unitriangular matrix with non-diagonal entries in $t^{2} \mathbb{Z}\left[t^{2}\right]$, so $\mathbf{D}_{d, n}(t)$ is invertible over $\mathbb{Z}\left[t^{2}, t^{-2}\right]$. Let $\mathbf{C}_{d, n}(t)=\left(c_{\mu \lambda}(t)\right)_{\lambda, \mu \in \Lambda^{+}(d, n)}$ be in the inverse of $\mathbf{D}_{d, n}(t)$. Then $\mathbf{C}_{d, n}(1)$ is in the inverse decomposition matrix of $\mathcal{S}_{q}(d, n), c_{\mu \lambda}(t) \in \mathbb{Z}\left[t^{2}, t^{-2}\right]$, and $c_{\mu \lambda}(1)=Q_{\mu \lambda}(-1)$ by (1.11). In fact, more is true. Since the $d_{\mu \lambda}(t)$ are parabolic Kazhdan-Lusztig polynomials, the $c_{\mu \lambda}(t)$ are inverse Kazhdan-Lusztig polynomials. Therefore, via the work of Varagnolo and Vasserot, the Lusztig conjecture implies that

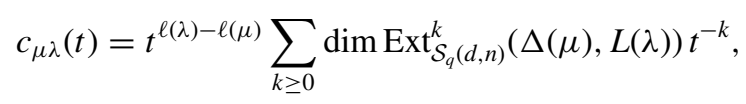

for a suitable length function $\ell: \Lambda^{+}(d, n) \rightarrow \mathbb{N}_{0}$; see [26, Section C2] or [4, Section 3.8]. In other words, $q^{\ell(\lambda)-\ell(\mu)} Q_{\mu \lambda}\left(t^{-1}\right)=c_{\mu \lambda}(t)$ is a renormalized parabolic Kazhdan-Lusztig polynomial.

Let us now apply these results in the case where $\lambda$ and $\mu$ admit a horizontal $s$-cut; that is, when $\lambda_{1}+\cdots+\lambda_{s}=\mu_{1}+\cdots+\mu_{s}$. By (1.10) we know that $Q_{\mu \lambda}=Q_{\mu^{t} \lambda^{t}} Q_{\mu^{b} \lambda^{b}}$. Therefore, it follows from (1.10) that $c_{\mu \lambda}(t)=c_{\mu^{t} \lambda^{t}}(t) c_{\mu^{b} \lambda^{b}}(t)$ - one can check that $\ell(\lambda)=\ell\left(\lambda^{t}\right)+\ell\left(\lambda^{b}\right)$, and similarly for $\ell(\mu)$. Consequently, because $\mathbf{D}_{d, n}(t)=\mathbf{C}_{d, n}(t)^{-1}$, we obtain the following. (Note that taking horizontal $s$-cuts is compatible with the dominance ordering.)

Corollary 1.12 (Chuang, Miyachi and Tan [3]) Suppose $\lambda_{1}+\cdots+\lambda_{s}=\mu_{1}+\cdots+\mu_{s}$. Then $d_{\mu \lambda}(t)=d_{\mu^{t} \lambda^{t}}(t) d_{\mu^{b} \lambda^{b}}(t)$.

In particular, by setting $t=1$ and applying Varagnolo and Vasserot's theorem [35], we recover Theorem 1.4. 
Chuang, Miyachi and Tan give a more elementary proof of this corollary using an inner product on the Fock space and the uniqueness of the canonical basis. Using a result of Leclerc [31], they also prove the column removal version of this result.

The last Corollary is interesting because, conjecturally [30,32], the coefficients of the polynomials $d_{\mu \lambda}(t)$ should describe the composition factors of the Jantzen filtrations of the two modules $\Delta(\mu)$ and $S(\lambda)$. Thus Corollary 1.12 suggests that, at least when $q \in \mathbb{C}$ is a root of unity, the Jantzen filtrations may also be invariant under row and column removal.

Corollary 1.12 can be viewed as a result for both the Hecke algebras and the $q$-Schur algebras. The astute reader will have noticed that we have not given a counterpart of (1.10) for Hecke algebras; that is, we have not given a row removal theorem for computing $\operatorname{Ext}_{\mathscr{H}}(S(\mu), D(\lambda))$. Although we have tried quite hard to prove this, we do not know whether or not the obvious analogue of (1.10) holds for Hecke algebras. We cannot repeat the argument used to prove Corollary 1.8 because (1.7), and more generally [14, Proposition 10.2], does not apply.

Finally we remark that some interesting additional results for the symmetric groups in characteristic $p>0$ have been proved by Hemmer [19,20]. Hemmer fixes a positive integer $m<n$ and considers the functor $\mathcal{F}_{m}$ which sends an $\mathfrak{S}_{n}$-module $M$ to its $\mathfrak{S}_{m}$ fixed points; so $\mathcal{F}_{m}(M)$ is naturally a $\mathfrak{S}_{n-m}$-module. Hemmer proves that if $\lambda$ is a partition of $n$ with $\lambda_{1}=m$ then $\mathcal{F}_{m}(S(\lambda)) \cong S\left(\lambda_{2}, \lambda_{3}, \ldots\right)$; that is, $\mathcal{F}_{m}(S(\lambda)) \cong S\left(\lambda^{b}\right)$ using the notation of Theorem 1.1 (with $s=1$ ). Hemmer also considers the effects of $\mathcal{F}_{m}$ on permutation modules and Young modules and he establishes row removal theorems for these modules as well.

Hemmer's work was motivated by trying to understand and prove Theorem 1 when $s=1$ for the symmetric groups using only the representation theory of the symmetric groups. In fact, Hemmer's functor is closely related to Donkin's truncation functor because Hemmer [19, Theorem 2.1] shows that $\mathcal{F}_{m}=\alpha \circ \operatorname{Tr}_{\Sigma}^{\lambda} \circ \beta$, for suitable Schur functors and inverse Schur functor with $\Sigma=\left\{\alpha_{2}, \ldots, \alpha_{n-1}\right\}$.

One of Hemmer's most interesting results is that when $\lambda_{1}=\mu_{1}=m<p$ there is an injection

$$
\operatorname{Ext}_{R}^{1} \mathfrak{S}_{n}(D(\lambda), D(\mu)) \hookrightarrow \operatorname{Ext}_{R}^{1} \mathfrak{S}_{n-m}\left(D\left(\lambda^{b}\right), D\left(\mu^{b}\right)\right),
$$

where $R$ is a field of characteristic $p$. The assumption that $m<p$ means that the functor $\mathcal{F}_{m}$ can be realized by multiplying by an idempotent in $R \mathfrak{S}_{n}$. Hemmer also uses results of Kleshchev and Sheth [29] on the cohomology of the symmetric groups. His argument would generalize to the Hecke algebras when $m<e$ if $q$-analogues of Kleshchev and Sheth's results were available.

\section{Definitions and preliminary results}

We begin by establishing some definitions. The notation used will be that of [33]. Recall the definitions of the Hecke algebra $\mathscr{H}=\mathscr{H}_{n}$ and the Schur algebra $\mathcal{S}=\mathcal{S}_{q}(d, n)$ from the introduction. 
Let $\lambda$ and $\mu$ be compositions of $n$. Say that $\lambda$ dominates $\mu$ and write $\lambda \unrhd \mu$ if

$$
\sum_{i=1}^{k} \lambda_{i} \geq \sum_{i=1}^{k} \mu_{i}
$$

for all $k$. If $\lambda \unrhd \mu$ and $\lambda \neq \mu$, we write $\lambda \triangleright \mu$.

Let $\lambda=\left(\lambda_{1}, \lambda_{2}, \ldots\right)$ be a composition of some integer $n$. The diagram of $\lambda$ is the set

$$
\left\{(i, j) \mid 1 \leq i \text { and } 1 \leq j \leq \lambda_{i}\right\}
$$

regarded as an array of nodes in the plane. A $\lambda$-tableau is obtained from the diagram of $\lambda$ by replacing each node with one of the integers $1,2, \ldots, n$, allowing no repeats. If $t$ is a $\lambda$-tableau we write Shape $(t)=\lambda$. A $\lambda$-tableau $t$ is row standard if its entries increase along the rows; and is standard if $\lambda$ is a partition and the entries of $t$ increase across the rows and down the columns. If $\lambda$ is a partition, define $\operatorname{Std}(\lambda)$ to be the set of standard $\lambda$-tableaux. The symmetric group $\mathfrak{S}_{n}$ acts from the right in a natural way on the set of tableaux.

Let $\lambda$ and $\mu$ be compositions of $n$. A $\lambda$-tableau of type $\mu$ is obtained by replacing each node in the diagram of $\lambda$ with one of the integers $1,2, \ldots$ such that the number $i$ occurs $\mu_{i}$ times. A $\lambda$-tableau $T$ (of type $\mu$ ) is semistandard if $\lambda$ is a partition, and the entries of $T$ are non-decreasing across the rows and strictly increasing down the columns. Let $\mathcal{T}_{0}(\lambda, \mu)$ denote the set of semistandard $\lambda$-tableaux of type $\mu$, and let $T^{\lambda}$ denote the unique semistandard $\lambda$-tableau of type $\lambda$.

Let $\lambda$ be a composition of $n$. Define $\mathfrak{t}^{\lambda}$ to be the standard $\lambda$-tableau in which the integers $1,2, \ldots, n$ are entered in increasing order along its rows, and $\mathfrak{S}_{\lambda}$ to be the row stabilizer of $\mathfrak{t}^{\lambda}$. Set

$$
m_{\lambda}=\sum_{\omega \in \mathfrak{S}_{\lambda}} T_{\omega} .
$$

We define the permutation module $M(\lambda)$ to be the right $\mathscr{H}$-module $M(\lambda)=m_{\lambda} \mathscr{H}$.

Definition 2.1 Suppose that $\lambda$ is a composition of $n$ and that $t$ is a row standard $\lambda$ tableau. Define $d(t) \in \mathfrak{S}_{n}$ to be such that $t=\mathfrak{t}^{\lambda} d(t)$. Now define ${ }^{*}$ to be the $R$-linear anti-automorphism of $\mathscr{H}$ determined by $T_{i}^{*}=T_{i}$. If $s, t$ are row standard $\lambda$-tableaux, define $m_{s t} \in \mathscr{H}$ by

$$
m_{s t}=T_{d(s)}^{*} m_{\lambda} T_{d(t)}
$$

Murphy [34] has shown that $\left\{m_{s t} \mid s, t \in \operatorname{Std}(\lambda)\right.$ for some partition $\lambda$ of $\left.n\right\}$ is a cellular basis of $\mathscr{H}$ in the sense of Graham and Lehrer [17]. Further, if $\mu$ is any composition of $n$ and $s, t$ are row standard $\mu$-tableaux then $m_{s t}$ can be written as a $R$-linear combination of $m_{u v}$ where $u, v \in \operatorname{Std}(\lambda)$, and $\lambda$ is a partition of $n$ such that $\lambda \unrhd \mu$.

Let $\lambda, \mu$ be compositions of $n$ and let $t$ be a $\lambda$-tableau. Define $\mu(t)$ to be the $\lambda$-tableau of type $\mu$ obtained by replacing each entry in $t$ with its row index in $\mathfrak{t}^{\mu}$. 
Definition 2.2 Suppose that $S$ is a semistandard $\lambda$-tableau of type $\mu$ and $t$ is a standard $\lambda$-tableau. Define

$$
m_{S t}=\sum_{s}\left\{m_{s t} \mid s \in \operatorname{Std}(\lambda) \text { and } \mu(s)=S\right\}
$$

As proved in [34], $M(\mu)$ is free as an $R$-module with basis

$$
\left\{m_{S t} \mid S \in \mathcal{T}_{0}(\lambda, \mu), t \in \operatorname{Std}(\lambda) \text { for some partition } \lambda \text { of } n\right\}
$$

Definition 2.3 Let $\lambda$ be a partition of $n$. Define $\mathscr{H}^{\lambda}$ to be the $R$-submodule of $\mathscr{H}$ with basis

$$
\left\{m_{s t} \mid s, t \in \operatorname{Std}(v) \text { where } v \unrhd \lambda\right\}
$$

and let $\breve{\mathscr{H}}^{\lambda}$ be the $R$-submodule of $\mathscr{H}$ with basis

$$
\left\{m_{s t} \mid s, t \in \operatorname{Std}(v) \text { where } v \triangleright \lambda\right\} .
$$

We define the Specht module $S(\lambda)$ to be the right $R$-module $\left(\breve{\mathscr{H}}^{\lambda}+m_{\lambda}\right) \mathscr{H}$.

It is shown in $[33,34]$ that $\mathscr{H}^{\lambda}$ and $\check{\mathscr{H}}^{\lambda}$ are both two-sided ideals of $\mathscr{H}$. It follows that $S(\lambda)$ is a right $\mathscr{H}$-module which is free as an $R$-module with basis $\left\{\breve{\mathscr{H}}^{\lambda}+m_{\mathfrak{t}^{\lambda}{ }_{t}} \mid t \in \operatorname{Std}(\lambda)\right\}$.

We now consider the $q$-Schur algebras $\mathcal{S}=\mathcal{S}_{q}(d, n)$ which we defined in the introduction. Fix a non-negative integer $d$ and for $\lambda \in \Lambda^{+}(d, n)$, set $\mathcal{T}_{0}(\lambda)=\bigcup_{\mu \in \Lambda(d, n)} \mathcal{T}_{0}(\lambda, \mu)$.

Definition 2.4 Let $\lambda, \mu \in \Lambda(d, n), v \in \Lambda^{+}(d, n)$ and suppose $S \in \mathcal{T}_{0}(v, \lambda), T \in$ $\mathcal{T}_{0}(\nu, \mu)$. If $\tau \in \Lambda(d, n)$, define $\varphi_{S T} \in \mathcal{S}$ by

$$
\varphi_{S T}\left(m_{\tau} h\right)=\delta_{\tau \mu} \sum_{s, t}\left\{m_{s t} h \mid s, t \in \operatorname{Std}(v) \text { and } \lambda(s)=S, \mu(t)=T\right\} \in M(\lambda)
$$

for all $h \in \mathscr{H}$. In fact $\left\{\varphi_{S T} \mid v \in \Lambda^{+}(d, n)\right.$ and $\left.S, T \in \mathcal{T}_{0}(v)\right\}$ is a cellular basis of $\mathcal{S}$ by [33, Theorem 4.13$]$.

Definition 2.5 Let $\lambda \in \Lambda^{+}(d, n), \mu \in \Lambda(d, n)$ and $T \in \mathcal{T}_{0}(\lambda, \mu)$. Define the map

$$
\varphi_{T}: \bigoplus_{\tau \in \Lambda(d, n)} M(\tau) \rightarrow S(\lambda)
$$

by

$$
\varphi_{T}\left(m_{\tau} h\right)=\delta_{\tau \mu} \sum_{t}\left\{\breve{\mathscr{H}}^{\lambda}+m_{\lambda} T_{d(t)} h \mid t \in \operatorname{Std}(\lambda) \text { and } \mu(t)=T\right\}
$$

for all $h \in \mathscr{H}$. 
By restriction, we will consider $\varphi_{T}$ to be an element of $\operatorname{Hom}_{\mathscr{H}}(M(\mu), S(\lambda))$.

Dipper and James [8, Corollary 8.7] have proved the next result for the family of Specht modules which are dual to the $S(\lambda)$. Our lemma is equivalent to the Dipper-James result.

Lemma 2.6 Let $\lambda$ and $\mu$ be partitions of $n$ and suppose that $q \neq-1$ or that $\mu$ is 2-restricted. Then $\operatorname{Hom}_{\mathscr{H}}(M(\mu), S(\lambda))$ is free as an $R$-module with basis $\left\{\varphi_{T} \mid T \in\right.$ $\left.\mathcal{T}_{0}(\lambda, \mu)\right\}$

Proof: First observe that if $\phi \in \operatorname{Hom}_{\mathscr{H}}(M(\mu), S(\lambda))$ then $\phi\left(m_{\mu} h\right)=\phi\left(m_{\mu}\right) h$, for all $h \in \mathscr{H}$, so $\phi$ is completely determined by the value of $\phi\left(m_{\mu}\right)$. In particular, the maps $\varphi_{T}$ are linearly independent because $\left\{\varphi_{T}\left(m_{\mu}\right) \mid T \in \mathcal{T}_{0}(\lambda, \mu)\right\}$ is linearly independent by the remark after Definition 2.3.

It remains to show that the set $\left\{\varphi_{T} \mid T \in \mathcal{T}_{0}(\lambda, \mu)\right\}$ spans $\operatorname{Hom}_{\mathscr{H}}\left(M^{\mu}, S^{\lambda}\right)$. Let $\phi \in$ $\operatorname{Hom}_{\mathscr{H}}\left(M^{\mu}, S^{\lambda}\right)$ and write $\phi\left(m_{\mu}\right)=\left\{\breve{H}^{\lambda}+\sum r_{t} m_{t^{\lambda} t} \mid t \in \operatorname{Std}(\lambda)\right\}$, for some $r_{t} \in R$. Fix a tableau $s$ such that $r_{s} \neq 0$ and $r_{t}=0$ whenever $s \triangleright t$. We claim that $\mu(s)$ is a semistandard tableau. If not, then there exist integers $i<j$ which are in the same column of $s$ such that $(i, j) \in \mathfrak{S}_{\mu}$. Now, $(j-1, j) \in \mathfrak{S}_{\mu}$ so $m_{\mu} T_{j}=q m_{\mu}$. Therefore

$$
\begin{aligned}
\check{\mathscr{H}}^{\lambda}+\sum r_{t} m_{t^{\lambda} t} & =\phi\left(m_{\mu}\right)=q^{-1} \phi\left(m_{\mu} T_{j-1}\right)=q^{-1} \phi\left(m_{\mu}\right) T_{j-1} \\
& =\breve{\mathscr{H}}^{\lambda}+\sum q^{-1} r_{t} m_{t^{\lambda} t} T_{j-1} .
\end{aligned}
$$

Let $s^{\prime}=s(j-1, j)$. If $i<j-1$ then $s^{\prime}$ is a standard tableau and $s$ dominates $s^{\prime}$, so $m_{t^{\lambda} s} T_{j-1}=m_{t^{\lambda} s^{\prime}}$, and $r_{s^{\prime}}=0$ by the choice of $s$. However, now ( $\dagger$ ) and [33, Corollary 3.9] show that $m_{t^{\lambda} s^{\prime}}$ appears with non-zero coefficient in $\phi\left(m_{\mu}\right)$, so this is a contradiction and we must have $i=j-1$. Therefore, $j-1$ and $j$ are in the same column of $s$ and so there exist $a_{v} \in R$ such that

$$
\breve{\mathscr{H}}^{\lambda}+m_{t^{\lambda} s} T_{j-1}=\breve{\mathscr{H}}^{\lambda}+-m_{t^{\lambda} s},+\sum\left\{a_{v} m_{t^{\lambda} v} \mid v \triangleright s\right\}
$$

by [33, Corollary 3.21]. Hence, comparing the coefficient of $m_{t^{\lambda} s}$ on both sides of ( $\dagger$ ) shows that $r_{s}=-q^{-1} r_{s}$. Since $q \neq-1$ we again have a contradiction. Hence, $\mu(s)$ is semistandard as claimed.

To complete the proof it is enough to show that $r_{t}=r_{t^{\prime}}$ whenever $\mu(t)=\mu\left(t^{\prime}\right)$. The proof of this is similar to [33, Proposition 3.18] and is left as an exercise for the reader.

Let $\mu \in \Lambda(d, n)$. Then $\varphi_{T^{\mu} T^{\mu}} \in \mathcal{S}$ restricts to the identity map $\varphi_{\mu}$ on $M(\mu)$, and $\varphi_{T^{\mu} T^{\mu}}$ is zero on $M(\tau)$ when $\tau \neq \mu$. Abusing notation slightly, we write $\varphi_{\mu}=\varphi_{T^{\mu} T^{\mu}}$.

Set $\mathcal{M}(\mu)$ to be the right $\mathcal{S}$-module

$$
\mathcal{M}(\mu)=\varphi_{\mu} \mathcal{S}
$$

Then $\mathcal{M}(\mu)$ has a basis given by $\left\{\varphi_{S T} \mid S \in \mathcal{T}_{0}(\lambda, \mu), T \in \mathcal{T}_{0}(\lambda)\right.$ where $\left.\lambda \in \Lambda^{+}(d, n)\right\}$. 
Definition 2.7 For $\lambda \in \Lambda^{+}(d, n)$, we define $\mathcal{S}^{\lambda}$ to be the two-sided ideal of $\mathcal{S}$ given by

$$
\mathcal{S}^{\lambda}=\left\{\varphi_{S T} \mid S, T \in \mathcal{T}_{0}(\nu) \text { where } v \in \Lambda^{+}(d, n) \text { and } \nu \unrhd \lambda\right\}
$$

and $\check{\mathcal{S}}^{\lambda}$ to be the two-sided ideal of $\mathcal{S}$ given by

$$
\breve{\mathcal{S}}^{\lambda}=\left\{\varphi_{S T} \mid S, T \in \mathcal{T}_{0}(v) \text { where } v \in \Lambda^{+}(d, n) \text { and } v \triangleright \lambda\right\} .
$$

We define the Weyl module $\Delta(\lambda)$ to be the right $\mathcal{S}$-module

$$
\Delta(\lambda)=\left(\check{\mathcal{S}}^{\lambda}+\varphi_{\lambda}\right) \mathcal{S} .
$$

Then $\Delta(\lambda)$ is free as an $R$-module with basis

$$
\left\{\check{\mathcal{S}}^{\lambda}+\varphi_{T^{\lambda} T} \mid T \in \mathcal{T}_{0}(\lambda)\right\} .
$$

In fact, we may identify $\check{\mathcal{S}}^{\lambda}+\varphi_{T^{\lambda} T}$ with the map $\varphi_{T} \in \operatorname{Hom}_{\mathscr{H}}\left(\oplus_{\tau} M(\tau), S(\lambda)\right)$ of Definition 2.5. Then $\Delta(\lambda)$ is isomorphic to the $\mathcal{S}$-submodule of $\operatorname{Hom}_{\mathscr{H}}\left(\oplus_{\tau} M(\tau), S(\lambda)\right)$ with basis $\left\{\varphi_{T} \mid T \in \mathcal{T}_{0}(\lambda)\right\}$. (Note that if $q \neq-1$ this is actually a basis of $\operatorname{Hom}_{\mathscr{H}}\left(\oplus_{\tau} M(\tau)\right.$, $S(\lambda)$ ) by Lemma 2.6.)

Definition 2.9 Let $\lambda \in \Lambda^{+}(d, n), \mu \in \Lambda(d, n)$ and $T \in \mathcal{T}_{0}(\lambda, \mu)$. Define $\theta_{T} \in$ $\operatorname{Hom}_{\mathcal{S}}(\mathcal{M}(\mu), \Delta(\lambda))$ by

$$
\theta_{T}\left(\varphi_{\mu} f\right)=\varphi_{T} f
$$

for all $f \in \mathcal{S}$.

Lemma 2.10 Suppose that $\lambda \in \Lambda^{+}(d, n)$ and $\mu \in \Lambda(d, n)$. Then $\left\{\theta_{T} \mid T \in \mathcal{T}_{0}(\lambda, \mu)\right\}$ is a basis of $\operatorname{Hom}_{\mathcal{S}}(\mathcal{M}(\mu), \Delta(\lambda))$.

Proof: Following the proof of Lemma 2.6, it is easy to show that if $\theta \in \operatorname{Hom}_{\mathcal{S}}(\mathcal{M}(\mu)$, $\Delta(\lambda)$ ), then $\theta$ is completely determined by the value of $\theta\left(\varphi_{\mu}\right)$, and that the maps $\left\{\theta_{T} \mid T \in\right.$ $\left.\mathcal{T}_{0}(\lambda, \mu)\right\}$ are linearly independent. In order to show that they span $\operatorname{Hom}_{\mathcal{S}}(\mathcal{M}(\mu), \Delta(\lambda))$, consider $\theta \in \operatorname{Hom}_{\mathcal{S}}(\mathcal{M}(\mu), \Delta(\lambda))$ such that $\theta\left(\varphi_{\mu}\right)=\left\{\check{\mathcal{S}}^{\lambda}+\sum r_{T} \varphi_{T^{\lambda} T} \mid T \in \mathcal{T}_{0}(\lambda)\right\}$ for some $r_{T} \in R$. Now note that $\varphi_{\mu} \varphi_{\nu}=0$ for $v \neq \mu$. Hence for $v \neq \mu$ we have that

$$
0=\theta\left(\varphi_{\mu} \varphi_{\nu}\right)=\left\{\check{\mathcal{S}}^{\lambda}+\sum r_{T} \varphi_{T^{\lambda} T} \mid T \in \mathcal{T}_{0}(\lambda, \nu)\right\}
$$

so that $r_{T}=0$ for $T \in \mathcal{T}_{0}(\lambda, \nu)$. This concludes the proof.

Classically, the next Proposition is a famous result of Carter and Lusztig [2]. The quantum analogue was first proved by Dipper and James [8]. The Dipper-James proof of this result is quite hard because they have to simultaneously construct a semistandard basis of the Weyl 
modules using what is essentially a Garnier relation argument. In contrast, from a cellular algebra perspective the Weyl modules come equipped with a semistandard basis, and this allows us to give a short bijective proof of this result.

Proposition 2.11 Let $\lambda$ and $\mu$ be partitions of $n$ and suppose that $q \neq-1$ or that $\mu$ is 2-restricted. Then

$$
\operatorname{Hom}_{\mathcal{S}}(\Delta(\mu), \Delta(\lambda)) \cong \operatorname{Hom}_{\mathscr{H}}(S(\mu), S(\lambda))
$$

as $R$-modules.

Proof: Suppose that $\theta \in \operatorname{Hom}_{\mathcal{S}}(\Delta(\mu), \Delta(\lambda))$. Let $\pi_{\mu}: \mathcal{M}(\mu) \rightarrow \Delta(\mu)$ be the natural projection map. Then $\theta \circ \pi_{\mu} \in \operatorname{Hom}_{\mathcal{S}}(\mathcal{M}(\mu), \Delta(\lambda))$ so we may write

$$
\theta \circ \pi_{\mu}=\sum_{T \in \mathcal{T}_{0}(\lambda, \mu)} r_{T} \theta_{T}
$$

for some $r_{T} \in R$. Let $\phi_{\theta}=\sum_{T} r_{T} \varphi_{T}$. Then $\phi_{\theta} \in \operatorname{Hom}_{\mathscr{H}}(M(\mu), S(\lambda))$ by Lemma 2.6. It is easy to see that $\phi_{\theta}\left(m_{\mu} h\right)=0$ whenever $m_{\mu} h \in \breve{\mathscr{H}}^{\mu}$, so we have a well-defined map $\dot{\theta}: S(\mu) \rightarrow S(\lambda)$ given by $\dot{\theta}\left[\left(\breve{\mathscr{H}}^{\mu}+m_{\mu}\right) h\right]=\phi_{\theta}\left(m_{\mu} h\right)$ for all $h \in \mathscr{H}$.

Conversely, suppose that $\phi \in \operatorname{Hom}_{\mathscr{H}}(S(\mu), S(\lambda))$. Let $p_{\mu}: M(\mu) \rightarrow S(\mu)$ be the natural projection map. Then $p_{\mu} \circ \phi \in \operatorname{Hom}_{\mathscr{H}}(M(\mu), S(\lambda))$. We can reverse the steps in the previous paragraph to define a map $\dot{\phi} \in \operatorname{Hom}_{\mathcal{S}}(\Delta(\mu), \Delta(\lambda))$.

As the two maps defined are mutually inverse bijections, the Proposition follows.

\section{A bijective proof of Theorem 1.2}

Recall that we have fixed a positive integer $s$. If $\lambda$ is a partition then the conjugate partition to $\lambda$ is the partition obtained by swapping the rows and columns of $\lambda$. Recall the definition of the partition $\lambda^{t}$ and $\lambda^{b}$ from the introduction. Using conjugation, we define analogous partition $\lambda^{l}$ and $\lambda^{r}$ as follows:

$$
\lambda^{l}=\left(\min \left(\lambda_{1}, s\right), \min \left(\lambda_{2}, s\right) \ldots,\right) \quad \lambda^{r}=\left(\max \left(\lambda_{1}-s, 0\right), \max \left(\lambda_{2}-s, 0\right) \ldots,\right)
$$

We recall the pictorial description of the partitions $\lambda^{t}, \lambda^{b}, \lambda^{l}$ and $\lambda^{r}$ :
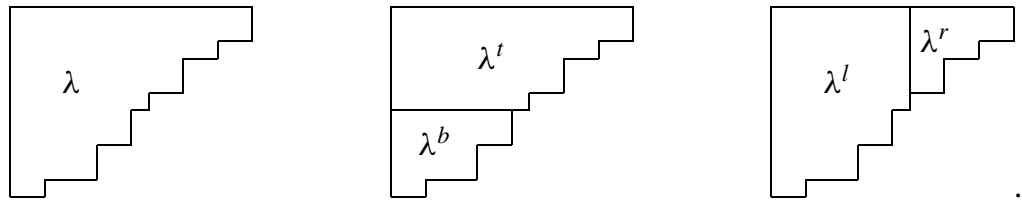

We can now state a stronger version of Theorem 1.2. 
Theorem 3.1 We fix positive integers $n$ and $d$, a commutative ring $R$ and an invertible element $q$ of $R$. For $\lambda, \mu \in \Lambda^{+}(d, n)$, we have the following results.

(i) Suppose that $\lambda_{1}+\cdots+\lambda_{s}=\mu_{1}+\cdots+\mu_{s}$. Then

$$
\begin{aligned}
& \operatorname{Hom}_{\mathcal{S}_{q}(d, n)}(\Delta(\mu), \Delta(\lambda)) \\
& \quad \cong \operatorname{Hom}_{\mathcal{S}_{q}\left(d^{\prime}, n-m\right)}\left(\Delta\left(\mu^{t}\right), \Delta\left(\lambda^{t}\right)\right) \otimes \operatorname{Hom}_{\mathcal{S}_{q}\left(d^{\prime \prime}, m\right)}\left(\Delta\left(\mu^{b}\right), \Delta\left(\lambda^{b}\right)\right) .
\end{aligned}
$$

where $m=\left|\lambda^{b}\right|$ and $d^{\prime}, d^{\prime \prime}>0$ satisfy $\mu^{t}, \lambda^{t} \in \Lambda^{+}\left(d^{\prime}, n-m\right)$ and $\mu^{b}, \lambda^{b} \in \Lambda^{+}\left(d^{\prime \prime}\right.$, $m)$.

(ii) Suppose that $\lambda_{1}^{\prime}+\cdots+\lambda_{s}^{\prime}=\mu_{1}^{\prime}+\cdots+\mu_{s}^{\prime}$. Then

$$
\begin{aligned}
& \qquad \operatorname{Hom}_{\mathcal{S}_{q}(d, n)}(\Delta(\lambda), \Delta(\mu)) \\
& \cong \operatorname{Hom}_{\mathcal{S}_{q}\left(d^{\prime}, n-m\right)}\left(\Delta\left(\lambda^{l}\right), \Delta\left(\mu^{l}\right)\right) \otimes \operatorname{Hom}_{\mathcal{S}_{q}\left(d^{\prime \prime}, m\right)}\left(\Delta\left(\lambda^{r}\right), \Delta\left(\mu^{r}\right)\right), \\
& \text { where } m=\left|\lambda^{r}\right| \text { and } d^{\prime}, d^{\prime \prime}>0 \text { satisfy } \mu^{l}, \lambda^{l} \in \Lambda^{+}\left(d^{\prime}, n-m\right) \text { and } \mu^{r}, \lambda^{r} \in \Lambda^{+}\left(d^{\prime \prime}, m\right) .
\end{aligned}
$$

Using a Ringel duality argument we will show below that both parts of the theorem are equivalent. We will prove part (i) by constructing explicit bijections between the two spaces of homomorphisms using the combinatorics of a cellular basis of $\mathcal{S}_{q}(d, n)$.

Applying (1.3) we immediately obtain the following more general version of Theorem 1.1.

Corollary 3.2 Let $\lambda$ and $\mu$ be partitions of n. Assume that $q \neq-1$ or that $\mu$ is 2 -restricted.

(i) Suppose that $\lambda_{1}+\cdots+\lambda_{s}=\mu_{1}+\cdots+\mu_{s}$. If $q=-1$, assume that $\mu^{t}$ is 2-restricted. Then

$$
\operatorname{Hom}_{\mathscr{H}_{n}}(S(\mu), S(\lambda)) \cong \operatorname{Hom}_{\mathscr{H}_{n-m}}\left(S\left(\mu^{t}\right), S\left(\lambda^{t}\right)\right) \otimes \operatorname{Hom}_{\mathscr{H}_{m}}\left(i\left(\mu^{b}\right), S\left(\lambda^{b}\right)\right) .
$$

where $m=\left|\lambda^{b}\right|$.

(ii) Suppose that $\lambda_{1}^{\prime}+\cdots+\lambda_{s}^{\prime}=\mu_{1}^{\prime}+\cdots+\mu_{s}^{\prime}$. Then

$$
\operatorname{Hom}_{\mathscr{H}_{n}}(S(\lambda), S(\mu)) \cong \operatorname{Hom}_{\mathscr{H}_{n-m}}\left(S\left(\lambda^{l}\right), S\left(\mu^{l}\right)\right) \otimes \operatorname{Hom}_{\mathscr{H}_{m}}\left(S\left(\lambda^{r}\right), S\left(\mu^{r}\right)\right),
$$

where $m=\left|\lambda^{r}\right|$.

Note that if $q=-1$ then Theorem 3.2 does not generally hold unless all partitions derived from $\mu$ are 2-restricted. For example, if $q=-1$ and $R$ is a field of characteristic 2 (so $\mathscr{H}_{n} \cong R \mathfrak{S}_{n}$ ) then

$$
\operatorname{Hom}_{\mathscr{H}_{2}}(S(1,1), S(1,1)) \cong R \cong \operatorname{Hom}_{\mathscr{H}_{2}}(S(2), S(1,1)),
$$

but $\operatorname{Hom}_{\mathscr{H}_{4}}(S(3,1), S(2,2))=0$.

We remark that it is possible to obtain an explicit bijection for the isomorphisms of Theorem 3.2(i) by mimicking the proof of Theorem 3.1(i) below.

We will first prove that parts (i) and (ii) of Theorem 3.1 are equivalent. The next lemma shows that we can reduce to the case where $d \geq n, d^{\prime} \geq n-l$ and $d^{\prime \prime} \geq l$. 
Lemma 3.3 Suppose that $\lambda, \mu \in \Lambda^{+}(d, n)$. Then

$$
\operatorname{Hom}_{\mathcal{S}_{q}(d, n)}(\Delta(\lambda), \Delta(\mu)) \cong \operatorname{Hom}_{\mathcal{S}_{q}(d+1, n)}(\Delta(\lambda), \Delta(\mu)) .
$$

Proof: This follows from the general theory of Schur functors, as can be found in [1, Section 3.1]. To see this let $\varphi_{d}=\sum\left\{\varphi_{\alpha} \mid \alpha \in \Lambda(d, n)\right\} \in \mathcal{S}_{q}(d+1, n)$. Then $\varphi_{d}$ is an idempotent so $P=\varphi_{d} \mathcal{S}_{q}(d+1, n)$ is a projective $\mathcal{S}_{q}(d+1, n)$-module. Since $\mathcal{S}_{q}(d, n) \cong$ $\varphi_{d} \mathcal{S}_{q}(d+1, n) \varphi_{d}$ we can define a functor

$$
\alpha: \operatorname{Mod}-\mathcal{S}_{q}(d+1, n) \rightarrow \operatorname{Mod}-\mathcal{S}_{q}(d, n) \quad \text { by } \quad \alpha=\operatorname{Hom}_{\mathcal{S}_{q}(d+1, n)}(P, \quad \text { ) } .
$$

If $\lambda, \mu \in \Lambda^{+}(d, n)$ then every composition factor of the $\mathcal{S}_{q}(d+1, n)$-modules $\Delta(\lambda)$ and $\Delta(\mu)$ are contained in the head of $P$, so $\operatorname{Hom}_{\mathcal{S}_{q}(d+1, n)}(\Delta(\lambda), \Delta(\mu)) \cong \operatorname{Hom}_{\mathcal{S}_{q}(d, n)}(\alpha(\Delta(\lambda))$, $\alpha(\Delta(\mu)))$ by [1, Corollary 3.1c]. It is easy to see that if $v \in \Lambda(d, n)$ then $\alpha(\Delta(\nu)) \cong \Delta(\nu)$, as $\mathcal{S}_{q}(d, n)$-modules, so this completes the proof.

To show that parts (i) and (ii) of Theorem 3.1 are equivalent we need some more notation. Fix an integer $d \geq n$ and for $\lambda \in \Lambda(d, n)$ define

$$
n_{\lambda}=\sum_{w \in \mathfrak{S}_{\lambda}}(-q)^{-\ell(w)} T_{w} .
$$

Set $N(\lambda)=n_{\lambda} \mathscr{H}$ and let $\mathcal{S}_{R}=\mathcal{S}_{R}(d, n)$ be the endomorphism algebra

$$
\mathcal{S}_{R}=\operatorname{End}_{\mathscr{H}}\left(\bigoplus_{\mu \in \Lambda(d, n)} N(\mu)\right) .
$$

Let \# : $\mathscr{H} \rightarrow \mathscr{H}$ be the $R$-linear map determined by $T_{w}^{\#}=(-q)^{-\ell(w)} T_{w^{-1}}^{-1}$, for all $w \in \mathfrak{S}_{n}$. Then it is easy to see that \# is an algebra automorphism of $\mathscr{H}$ and that $n_{\mu}^{\#}=m_{\mu}$, for all $\mu \in \Lambda(d, n)$; see, for example, [6]. Therefore, $N(\mu)=(M(\mu))^{\#}$ and $\operatorname{Hom}_{\mathscr{H}}(M(\mu), M(\lambda)) \cong$ $\operatorname{Hom}_{\mathscr{H}}(N(\mu), N(\lambda))$. Consequently, \# induces a canonical algebra isomorphism $F: \mathcal{S}_{q}(d, n) \stackrel{\simeq}{\rightarrow} \mathcal{S}_{R}(d, n)$; explicitly, $F(\phi)(h)=\left(\phi\left(h^{\#}\right)\right)^{\#}$, for all $\phi \in \mathcal{S}_{q}(d, n)$ and all $h \in \mathscr{H}$. For $\lambda \in \Lambda^{+}(d, n)$ define $\Delta(\lambda)_{R}=F(\Delta(\lambda))$. Then $\Delta(\lambda)_{R}$ is an analogue of a Weyl module for the algebra $\mathcal{S}_{R}(d, n)$.

Donkin has shown that if $d \geq n$ then $\mathcal{S}_{R}(d, n)$ is the Ringel dual of the $q$-Schur algebra $\mathcal{S}_{q}(d, n)$ [13]. Consequently, we have a contravariant functor $G$ which sends $\mathcal{S}_{q}(d, n)$ modules to $\mathcal{S}_{R}(d, n)$-modules. Further, if $v \in \Lambda(d, n)$ then $G(\Delta(v)) \cong \Delta_{R}\left(v^{\prime}\right)^{\diamond}$, where $\Delta_{R}\left(v^{\prime}\right)^{\diamond}$ is the contragredient dual of $\Delta_{R}\left(v^{\prime}\right)$. Proofs and further explanations of these results can be found in [13, Sections 4.1 and A4].

Lemma 3.4 Suppose that $d \geq n$ and $\lambda, \mu \in \Lambda^{+}(d, n)$ Then

$$
\operatorname{Hom}_{\mathcal{S}_{q}(d, n)}(\Delta(\mu), \Delta(\lambda)) \cong \operatorname{Hom}_{\mathcal{S}_{q}(d, n)}\left(\Delta\left(\lambda^{\prime}\right), \Delta\left(\mu^{\prime}\right)\right)
$$


Proof: $\quad$ Since $d \geq n, \mathcal{S}_{R}(d, n)$ is the Ringel dual of $\mathcal{S}_{q}(d, n)$. Therefore, using the functors above, we have

$$
\begin{aligned}
\operatorname{Hom}_{\mathcal{S}_{q}(d, n)}(\Delta(\mu), \Delta(\lambda)) & \cong \operatorname{Hom}_{\mathcal{S}_{R}(d, n)}\left(\Delta_{R}(\mu), \Delta_{R}(\lambda)\right), \quad \text { by applying } F, \\
& \cong \operatorname{Hom}_{\mathcal{S}_{R}(d, n)}\left(\Delta_{R}(\lambda)^{\diamond}, \Delta_{R}(\mu)^{\diamond}\right), \quad \text { by duality, } \\
& \cong \operatorname{Hom}_{\mathcal{S}_{q}(d, n)}\left(\Delta\left(\lambda^{\prime}\right), \Delta\left(\mu^{\prime}\right)\right),
\end{aligned}
$$

where the last line follows by using $G$.

Remark If $X$ and $Y$ are $\mathcal{S}_{q}(d, n)$-modules which both have a $\nabla$-filtration then $\operatorname{Ext}_{\mathcal{S}_{q}(d, n)}^{k}$ $(X, Y) \cong \operatorname{Ext}_{\mathcal{S}_{R}(d, n)}^{k}(G(X), G(Y))$ by [13, Section A2.2]. Hence, $\operatorname{Ext}_{\mathcal{S}_{q}(d, n)}^{k}(\Delta(\mu), \Delta(\lambda)) \cong$ $\operatorname{Ext}_{\mathcal{S}_{q}(d, n)}^{k}\left(\Delta\left(\lambda^{\prime}\right), \Delta\left(\mu^{\prime}\right)\right)$, for all $k \geq 0$ by the argument above. This result is due to Donkin $[13,4.1 .5]$.

Proposition 3.5 Theorem 3.1(i) holds if and only if Theorem 3.1(ii) holds.

Proof: By Lemma 3.3 we may assume that $d, d^{\prime}, d^{\prime \prime}$ are all large enough so that we can apply Lemma 3.4.

If $\lambda$ and $\mu$ satisfy the conditions of Theorem 3.1(i), then $\lambda^{\prime}$ and $\mu^{\prime}$ satisfy the conditions of Theorem 3.1(ii), with $r=s, l=m$ and with

$$
\begin{aligned}
& \left(\lambda^{\prime}\right)^{l}=\left(\lambda^{t}\right)^{\prime}, \quad\left(\lambda^{\prime}\right)^{r}=\left(\lambda^{b}\right)^{\prime}, \\
& \left(\mu^{\prime}\right)^{l}=\left(\mu^{t}\right)^{\prime}, \quad\left(\mu^{\prime}\right)^{r}=\left(\mu^{b}\right)^{\prime} .
\end{aligned}
$$

By Lemma 3.4 we have $\operatorname{Hom}_{\mathcal{S}_{q}(d, n)}(\Delta(\mu), \Delta(\lambda)) \cong \operatorname{Hom}_{\mathcal{S}_{q}(d, n)}\left(\Delta\left(\lambda^{\prime}\right), \Delta\left(\mu^{\prime}\right)\right)$ and

$$
\begin{aligned}
& \operatorname{Hom}_{\mathcal{S}_{q}\left(d^{\prime}, n-m\right)}\left(\Delta\left(\mu^{t}\right), \Delta\left(\lambda^{t}\right)\right) \otimes \operatorname{Hom}_{\mathcal{S}_{q}\left(d^{\prime \prime}, m\right)}\left(\Delta\left(\mu^{b}\right), \Delta\left(\lambda^{b}\right)\right) \\
& \quad \cong \operatorname{Hom}_{\mathcal{S}_{q}\left(d^{\prime}, n-m\right)}\left(\Delta\left(\left(\lambda^{\prime}\right)^{l}\right), \Delta\left(\left(\mu^{\prime}\right)^{l}\right)\right) \otimes \operatorname{Hom}_{\mathcal{S}_{q}\left(d^{\prime \prime}, m\right)}\left(\Delta\left(\left(\lambda^{\prime}\right)^{r}\right), \Delta\left(\left(\mu^{\prime}\right)^{r}\right)\right) .
\end{aligned}
$$

The proof of the converse is similar.

We now prove Theorem 3.1. Fix integers $n, m, d, d^{\prime}$ and $d^{\prime \prime}$ as in the statement of the theorem and fix partitions $\lambda, \mu \in \Lambda^{+}(d, n)$ such that

$$
\lambda_{1}+\cdots+\lambda_{s}=\mu_{1}+\cdots+\mu_{s}=n-m,
$$

and $\lambda^{t}, \mu^{t} \in \Lambda^{+}\left(d^{\prime}, n-m\right)$, and $\lambda^{b}, \mu^{b} \in \Lambda^{+}\left(d^{\prime \prime}, m\right)$.

We need to define Schur algebras corresponding to the partitions $\lambda^{t}$ and $\lambda^{b}$. First, recall that $\mathscr{H}=\mathscr{H}_{n}$ is the Hecke algebra of $\mathfrak{S}_{n}$. It is the algebra generated by $T_{1}, T_{2}, \ldots, T_{n-1}$ subject to the relations given in the introduction. Define $\mathscr{H}^{t} \cong \mathscr{H}_{n-m}$ to be the quantum subgroup of $\mathscr{H}$ generated by

$$
T_{1}, T_{2}, \ldots, T_{n-m-1} \text {. }
$$


Similarly, define $\mathscr{H}^{b} \cong \mathscr{H}_{m}$ to be the quantum subgroup of $\mathscr{H}$ generated by

$$
T_{n-m+1}, T_{n-m+2}, \ldots, T_{n-1} .
$$

Note that if $h^{t} \in \mathscr{H}^{t}$ and $h^{b} \in \mathscr{H}^{b}$ then $h^{t} h^{b}=h^{b} h^{t}$.

By definition, $\mathfrak{S}_{n-m}$ is the full group of permutations acting on the set $\{1,2, \ldots, n-m\}$. Similarly, define $\mathfrak{S}_{m}^{b}$ to be the permutation group acting on $\{n-m+1, n-m+2, \ldots, n\}$. Then $\mathscr{H}^{t} \cong \mathscr{H}_{R, q}\left(\mathfrak{S}_{n-m}\right)$ and $\mathscr{H}^{b} \cong \mathscr{H}_{R, q}\left(\mathfrak{S}_{m}^{b}\right)$.

Suppose that $v$ is any partition of $n$ such that $v_{1}+v_{2}+\cdots+v_{s}=n-m$. Then $\mathfrak{t}^{v^{t}}$ is the standard $v^{t}$-tableau with entries $1,2, \ldots, n-m$ increasing in order along the rows. Similarly, define $\mathfrak{t}^{\nu^{b}}$ to be the standard $v^{b}$-tableau with entries $n-m+1, n-m+2, \ldots, n$ increasing in order along the rows. Then we also have corresponding subgroups $\mathfrak{S}_{v^{t}} \subseteq \mathfrak{S}_{n-m}$ and $\mathfrak{S}_{v^{b}}^{b} \subseteq \mathfrak{S}_{m}^{b}$ which are the row stabilizers of the tableaux $\mathfrak{t}^{t^{t}}$ and $\mathfrak{t}^{\mathfrak{w}^{b}}$ respectively. Now define

$$
m_{v^{t}}=\sum_{\omega \in \mathfrak{S}_{v^{t}}} T_{\omega} \in \mathscr{H}^{t} \quad \text { and } \quad m_{v^{b}}=\sum_{\omega \in \mathfrak{S}_{v^{b}}^{b}} T_{\omega} \in \mathscr{H}^{b} .
$$

Note that $m_{v}=m_{v^{t}} m_{v^{b}}=m_{v^{b}} m_{v^{t}}$ in $\mathscr{H}$. Then we have

$$
M\left(v^{t}\right)=m_{v^{t}} \mathscr{H}^{t} \quad \text { and } \quad M\left(v^{b}\right)=m_{v^{b}} \mathscr{H}^{b} .
$$

Since $\mathscr{H}^{t} \cong \mathscr{H}_{n-m}$ and $\mathscr{H}^{b} \cong \mathscr{H}_{m}$ we can use Definition 2.1 to define cellular bases of $\mathscr{H}^{t}$ and $\mathscr{H}^{b}$ in the obvious way. So, the tableaux indexing the cellular basis of $\mathscr{H}^{t}$ contain as entries the numbers $1, \ldots, n-m$, whereas the tableaux indexing the basis of $\mathscr{H}^{b}$ contain $n-m+1, \ldots, n$. Using these bases we define ideals $\breve{\mathscr{C}}^{v^{t}}$ and $S\left(v^{t}\right)$ of $\mathscr{H}^{t}$ and ideals $\breve{\mathscr{H}}{ }^{\nu^{b}}$ and $S\left(v^{b}\right)$ of $\mathscr{H}^{b}$.

Recall that $\mathcal{S}=\operatorname{End}_{\mathscr{H}}\left(\oplus_{\tau \in \Lambda(\mathrm{d}, \mathrm{n})} M(\tau)\right)$. We similarly define

$$
\mathcal{S}^{t}=\operatorname{End}_{\mathscr{H}}\left(\bigoplus_{\tau \in \Lambda\left(d^{\prime}, n-m\right)} M(\tau)\right) \quad \text { and } \quad \mathcal{S}^{\mathrm{b}}=\operatorname{End}_{\mathscr{H} p^{b}}\left(\bigoplus_{\tau \in \Lambda\left(d^{\prime \prime}, m\right)} M(\tau)\right) \text {. }
$$

Then $\mathcal{S}^{t} \cong \mathcal{S}_{q}\left(d^{\prime}, n-m\right)$ and $\mathcal{S}^{b} \cong \mathcal{S}_{q}\left(d^{\prime \prime}, m\right)$. Consequently, we have natural cellular algebras for both of these algebras and analogous modules $\check{\mathcal{S}}^{v^{t}}, \Delta\left(v^{t}\right)$ and so on.

Recall from Lemma 2.10 that $\operatorname{Hom}_{\mathcal{S}}(\mathcal{M}(\mu), \Delta(\lambda))$ has basis $\left\{\theta_{A} \mid A \in \mathcal{T}_{0}(\lambda, \mu)\right\}$, where $\theta_{A}$ is the map $\theta_{A}\left(\varphi_{\mu} f\right)=\varphi_{A} f$, for all $f \in \mathcal{S}$. We have similar bases for $\operatorname{Hom}_{\mathcal{S}^{t}}\left(\mathcal{M}\left(\mu^{t}\right)\right.$, $\left.\Delta\left(\lambda^{t}\right)\right)$ and $\operatorname{Hom}_{\mathcal{S}^{b}}\left(\mathcal{M}\left(\mu^{b}\right), \Delta\left(\lambda^{b}\right)\right)$.

Before we can begin the proof of Theorem 3.1 we need the following combinatorial definition.

Definition 3.6 Let $A^{t} \in \mathcal{T}_{0}\left(\lambda^{t}, \mu^{t}\right)$ and $A^{b} \in \mathcal{T}_{0}\left(\lambda^{b}, \mu^{b}\right)$ be two semistandard tableaux and suppose that $A^{t} \in \mathcal{T}_{0}\left(\lambda^{t}, \mu^{t}\right)$ has entry $x_{j}^{i}$ in position $i$ of row $j$ and $A^{b} \in \mathcal{T}_{0}\left(\lambda^{b}, \mu^{b}\right)$ has entry $y_{j}^{i}$ in position $i$ of row $j$. Define $\left(A^{t}, A^{b}\right)$ to be the $\lambda$-tableau of type $\mu$ with entry 
$z_{j}^{i}$ in position $i$ of row $j$, where

$$
z_{j}^{i}=\left\{\begin{array}{ll}
x_{j}^{i} & (j \leq s) \\
y_{j}^{i}+s & (j>s)
\end{array} .\right.
$$

For example, if

$$
\begin{aligned}
& \begin{array}{lllllllll}
1 & 1 & 1 & 1 & 1 & 2 & 1 & 1 & 2
\end{array} \\
& A^{t}=\begin{array}{lllll}
2 & 2 & 2 & 3
\end{array} \quad, \quad A^{b}=2 \quad 3 \quad \text {, } \\
& \begin{array}{llll}
3 & 3 & 3 & 3
\end{array}
\end{aligned}
$$

then

$$
\left(A^{t}, A^{b}\right)=\begin{array}{llllll}
1 & 1 & 1 & 1 & 1 & 2 \\
2 & 2 & 2 & 3 & & \\
3 & 3 & 3 & & & \\
4 & 4 & 5 & & & \\
5 & 6 & & & & \\
6 & & & & &
\end{array} .
$$

\section{Lemma 3.7}

(i) Suppose that $A^{t} \in \mathcal{T}_{0}\left(\lambda^{t}, \mu^{t}\right)$ and $A^{b} \in \mathcal{T}_{0}\left(\lambda^{b}, \mu^{b}\right)$. Then $\left(A^{t}, A^{b}\right)$ is a semistandard $\lambda$-tableau of type $\mu$.

(ii) $\mathcal{T}_{0}(\lambda, \mu)=\left\{\left(A^{t}, A^{b}\right) \mid A^{t} \in \mathcal{T}_{0}\left(\lambda^{t}, \mu^{t}\right)\right.$ and $\left.A^{b} \in \mathcal{T}_{0}\left(\lambda^{b}, \mu^{b}\right)\right\}$.

Proof: The first statement is clear. For (ii), suppose that $A$ is a semistandard $\lambda$-tableau of type $\mu$ with entry $z_{j}^{i}$ in position $i$ of row $j$. Then the entries of $A$ strictly increase down the columns, so that for $j>s$ we have that $z_{j}^{i}>s$. Since the number, $n-m$, of entries less than or equal to $s$ equals the number of positions in rows $1, \ldots, s$, it also is also true that $z_{j}^{i} \leq s$ for $j \leqslant s$. So the top $s$ rows of $A$ constitute a $\lambda^{t}$-tableau of type $\mu^{t}$ (which is clearly semistandard), and a similar statement holds for the lower rows.

Recall our convention from above that the standard $v^{b}$-tableaux contain the numbers $n-m+1, \ldots, n$ as entries. As an immediate corollary we obtain the following.

Corollary 3.8 Suppose that $A \in \mathcal{T}_{0}(\nu, \mu)$. Then

$$
\begin{aligned}
& \{a \mid a \in \operatorname{Std}(v) \text { and } \mu(a)=A\} \\
& \quad=\left\{\left(a^{t}, a^{b}\right) \mid a^{t} \in \operatorname{Std}\left(v^{t}\right) \text { and } \mu^{t}\left(\mathrm{a}^{t}\right)=\mathrm{A}^{t}, \mathrm{a}^{\mathrm{b}} \in \operatorname{Std}\left(v^{\mathrm{b}}\right) \text { and } \mu^{b}\left(a^{b}\right)=A^{b}\right\} .
\end{aligned}
$$


The key definition that we need to prove Theorem 3.1 is the following.

Definition 3.9 Suppose that $\theta^{t} \in \operatorname{Hom}_{\mathcal{S}^{t}}\left(\mathcal{M}\left(\mu^{t}\right), \Delta\left(\lambda^{t}\right)\right)$ and $\theta^{b} \in \operatorname{Hom}_{\mathcal{S}^{b}}\left(\mathcal{M}\left(\mu^{b}\right)\right.$, $\left.\Delta\left(\lambda^{b}\right)\right)$, where

$$
\theta^{t}=\sum_{A^{t}}\left\{\gamma_{A^{t}} \theta_{A^{t}} \mid A^{t} \in \mathcal{T}_{0}\left(\lambda^{t}, \mu^{t}\right)\right\} \in \operatorname{Hom}_{\mathcal{S}^{t}}\left(\mathcal{M}\left(\mu^{t}\right), \Delta\left(\lambda^{t}\right)\right)
$$

and

$$
\theta^{b}=\sum_{A^{b}}\left\{\gamma_{A^{b}} \theta_{A^{b}} \mid A^{b} \in \mathcal{T}_{0}\left(\lambda^{b}, \mu^{b}\right)\right\} \in \operatorname{Hom}_{\mathcal{S}^{b}}\left(\mathcal{M}\left(\mu^{b}\right), \Delta\left(\lambda^{b}\right)\right)
$$

Define the map $\theta=\left(\theta^{t}, \theta^{b}\right): \mathcal{M}(\mu) \rightarrow \Delta(\lambda)$ by $\theta=\sum_{A^{t}} \sum_{A^{b}} \gamma_{A^{t}} \gamma_{A^{b}} \theta_{\left(A^{t}, A^{b}\right)}$.

Note that by Lemma 2.10, this defines a bijection

$$
\operatorname{Hom}_{\mathcal{S}^{t}}\left(\mathcal{M}\left(\mu^{t}\right), \Delta\left(\lambda^{t}\right)\right) \otimes \operatorname{Hom}_{\mathcal{S}^{b}}\left(\mathcal{M}\left(\mu^{b}\right), \Delta\left(\lambda^{b}\right)\right) \rightarrow \operatorname{Hom}_{\mathcal{S}}(\mathcal{M}(\mu), \Delta(\lambda))
$$

We need two simple lemmas before we can exploit this observation.

Lemma 3.10 Suppose that $\lambda \unrhd v \triangleright \mu$. Then $v_{1}+\cdots+v_{s}=n-m$ and either $v^{t} \triangleright \mu^{t}$ or $v^{b} \triangleright \mu^{b}$.

Proof: The proof follows directly from the definition of $\unrhd$.

Lemma 3.11 Suppose that $v \triangleright \mu$ and that $S \in \mathcal{T}_{0}(\nu, \mu)$. Let

$$
m=\sum_{s}\left\{T_{d(s)}^{*} m_{\nu} \mid s \in \operatorname{Std}(v) \text { and } \mu(s)=S\right\}
$$

Then $m=m_{\mu} h^{t} h^{b}$ for some $h^{t}, h^{b}$ such that $h^{t} \in \mathscr{H}^{t}$ and $h^{b} \in \mathscr{H}^{b}$ and either $m_{\mu^{t}} h^{t} \in \breve{\mathscr{H}}^{\mu^{t}}$ or $m_{\mu^{b}} h^{b} \in \check{\mathscr{H}}^{\mu^{b}}$.

Proof: Define

$$
m^{t}=\sum_{s^{t}}\left\{T_{d\left(s^{t}\right)}^{*} m_{v^{t}} \mid s^{t} \in \operatorname{Std}\left(v^{t}\right) \text { and } \mu^{t}\left(s^{t}\right)=S^{t}\right\} .
$$

Then $m^{t} \in M\left(\mu^{t}\right)$ and so $m^{t}=m_{\mu^{t}} h^{t}$ for some $h^{t} \in \mathscr{H}^{t}$. Similarly, we can define

$$
m^{b}=\sum_{s^{b}}\left\{T_{d\left(s^{b}\right)}^{*} m_{v^{b}} \mid s^{b} \in \operatorname{Std}\left(v^{b}\right) \text { and } \mu^{b}\left(s^{b}\right)=S^{b}\right\} .
$$


so that $m^{b}=m_{\mu^{b}} h^{b}$ for some $h^{b} \in \mathscr{H}^{b}$. If $v^{t} \triangleright \mu^{t}$ then $m_{\mu^{t}} h^{t} \in \check{\mathscr{H}}^{\mu^{t}}$; otherwise, by Lemma 3.10, $v^{b} \triangleright \mu^{b}$ and $m_{\mu^{b}} h^{b} \in \check{\mathscr{C}}^{\mu^{b}}$. Then

$$
\begin{aligned}
m= & \sum_{s}\left\{T_{d(s)}^{*} m_{v} \mid s \in \operatorname{Std}(v) \text { and } \mu(s)=S\right\} \\
= & \sum_{s^{t}} \sum_{s^{b}}\left\{T_{d\left(s^{t}\right)}^{*} T_{d\left(s^{b}\right)}^{*} m_{v^{t}} m_{v^{b}} \mid s^{t} \in \operatorname{Std}\left(v^{t}\right) \text { and } \mu^{t}\left(s^{t}\right)\right. \\
& \left.=S^{t}, s^{b} \in \operatorname{Std}\left(v^{b}\right) \text { and } \mu^{b}\left(s^{b}\right)=S^{b}\right\}
\end{aligned}
$$

by Lemma 3.8. Therefore, $m=m^{t} m^{b}=m_{\mu^{t}} h^{t} m_{\mu^{b}} h^{b}=m_{\mu} h^{t} h^{b}$, completing the proof.

Proposition 3.12 Let $v \in \Lambda^{+}(d, n)$ and $\tau \in \Lambda(d, n)$ be partitions such that $\lambda \unrhd v \triangleright \mu$. Suppose that $U \in \mathcal{T}_{0}(\nu, \mu), V \in \mathcal{T}_{0}(\nu, \tau)$ and $A \in \mathcal{T}_{0}(\lambda, \mu)$. Then $\theta_{A}\left(\varphi_{U V}\right)=0$.

Proof: Define $\theta_{T^{\lambda} A} \in \operatorname{Hom}_{\mathcal{S}}(\mathcal{M}(\mu), \mathcal{M}(\lambda))$ by $\theta_{T^{\lambda} A}\left(\varphi_{\mu} f\right)=\varphi_{T^{\lambda} A} f$, for all $f \in \mathcal{S}$. Then $\theta_{T^{\lambda} A}$ is a well defined $\mathcal{S}$-homomorphism and $\theta_{A}=\pi \circ \theta_{T^{\lambda} A}$ where $\pi: \mathcal{M}(\lambda) \rightarrow \Delta(\lambda)$ is the natural projection. Note that $\varphi_{U V}=\varphi_{\mu} \varphi_{U V}$. Then

$$
\theta_{T^{\lambda} A}\left(\varphi_{U V}\right)=\left(\theta_{T^{\lambda} A}\left(\varphi_{\mu}\right)\right) \varphi_{U V}=\varphi_{T^{\lambda} A} \varphi_{U V} .
$$

Now $\varphi_{T^{\lambda} A} \in \mathcal{S}^{\lambda}$ and $\varphi_{U V} \in \mathcal{S}^{\nu}$. So, since $\lambda \unrhd v$, we have that $\varphi_{T^{\lambda} A} \varphi_{U V} \in \check{\mathcal{S}}^{\lambda}$. Hence $\theta_{A}\left(\varphi_{U V}\right)=\pi\left(\varphi_{T^{\lambda} A} \varphi_{U V}\right)=0$.

Proposition 3.13 Let $v$ and $\tau$ be partitions in $\Lambda^{+}(d, n)$ with $\lambda \unrhd v \triangleright \mu$. Suppose that

$$
\theta^{t} \in \operatorname{Hom}_{\mathcal{S}^{t}}\left(\mathcal{M}\left(\mu^{t}\right), \Delta\left(\lambda^{t}\right)\right) \quad \text { and } \quad \theta^{b} \in \operatorname{Hom}_{\mathcal{S}^{b}}\left(\mathcal{M}\left(\mu^{b}\right), \Delta\left(\lambda^{b}\right)\right)
$$

are two maps such that

$$
\theta^{t}\left(\varphi_{\mu^{t}} f\right)=0 \quad \text { whenever } f \in \mathcal{S}^{t} \text { and } \varphi_{\mu^{t}} f \in \check{\mathcal{S}}^{\mu^{t}}
$$

and

$$
\theta^{b}\left(\varphi_{\mu^{b}} f\right)=0 \quad \text { whenever } f \in \mathcal{S}^{b} \text { and } \varphi_{\mu^{b}} f \in \check{\mathcal{S}}^{\mu^{b}} .
$$

Let $\theta=\left(\theta^{t}, \theta^{b}\right)$ as in Definition 3.9 and suppose that $U \in \mathcal{T}_{0}(\nu, \mu)$ and $V \in \mathcal{T}_{0}(\nu, \tau)$. Then

$$
\theta\left(\varphi_{U V}\right)=0 .
$$

Proof: Write

$$
\theta^{t}=\sum_{A^{t}}\left\{\gamma_{A^{t}} \theta_{A^{t}} \mid A^{t} \in \mathcal{T}_{0}\left(\lambda^{t}, \mu^{t}\right)\right\} \quad \text { and } \quad \theta^{b}=\sum_{A^{b}}\left\{\gamma_{A^{b}} \theta_{A^{b}} \mid A^{b} \in \mathcal{T}_{0}\left(\lambda^{b}, \mu^{b}\right)\right\} .
$$


Note that $\varphi_{U V}=\varphi_{\mu} \varphi_{U V}$. Then

$$
\begin{aligned}
\theta\left(\varphi_{U V}\right) & =\sum_{A^{t}} \sum_{A^{b}} \gamma_{A^{t}} \gamma_{A^{b}} \theta_{\left(A^{t}, A^{b}\right)}\left(\varphi_{\mu}\right) \varphi_{U V} \\
& =\sum_{A^{t}} \sum_{A^{b}} \gamma_{A^{t}} \gamma_{A^{b}} \varphi_{\left(A^{t}, A^{b}\right)} \varphi_{U V} \in \operatorname{Hom}_{\mathscr{H}}(M(\tau), S(\lambda)) .
\end{aligned}
$$

Then, using the notation of Lemma 3.11, there exist $h^{t} \in \mathscr{H}^{t}, h^{b} \in \mathscr{H}^{b}$ such that

$$
\begin{aligned}
{\left[\theta\left(\varphi_{U V}\right)\right]\left(m_{\tau}\right)=} & \sum_{A^{t}} \sum_{A^{b}} \gamma_{A^{t}} \gamma_{A^{b}} \varphi_{\left(A^{t}, A^{b}\right)}\left(\varphi_{U V}\left(m_{\tau}\right)\right) \\
= & \sum_{A^{t}} \sum_{A^{b}} \gamma_{A^{t}} \gamma_{A^{b}} \varphi_{\left(A^{t}, A^{b}\right)} \\
& \times\left(\sum_{u} \sum_{v}\left\{T_{d(u)}^{*} m_{v} T_{d(v)} \mid u, v \in \operatorname{Std}(v), \mu(u)=U, \tau(v)=V\right\}\right) \\
= & \sum_{A^{t}} \sum_{A^{b}} \gamma_{A^{t}} \gamma_{A^{b}} \varphi_{\left(A^{t}, A^{b}\right)}\left(m_{\mu} h^{t} h^{b} \sum_{v} T_{d(v)}\right) \\
= & \check{\mathscr{H}}^{\lambda}+\sum_{A^{t}} \sum_{A^{b}} \sum_{a^{t}} \sum_{a^{b}}\left\{\gamma_{A^{t}} \gamma_{A^{b}} m_{\lambda^{t}} m_{\lambda^{b}} T_{d\left(a^{t}\right)} T_{d\left(a^{b}\right)} h^{t} h^{b} \sum_{v} T_{d(v)}\right. \\
& \left.\mid a^{t} \in \operatorname{Std}\left(\lambda^{t}\right), a^{b} \in \operatorname{Std}\left(\lambda^{b}\right), \mu^{t}\left(a^{t}\right)=A^{t}, \mu^{b}\left(a^{b}\right)=A^{t}\right\}
\end{aligned}
$$

wthere either $v^{t} \triangleright \mu^{t}$ and $m_{\mu^{t}} h^{t} \in \check{\mathscr{H}} \mu^{\mu^{t}}$ or $\nu^{b} \triangleright \mu^{b}$ and $m_{\mu^{b}} h^{b} \in \check{\mathscr{H}}^{\mu^{b}}$. Without loss of generality, assume $m_{\mu^{t}} h^{t} \in \check{\mathscr{H}} \mu^{\mu^{t}}$. Consider $\varphi_{U^{t} T^{v^{t}}} \in \operatorname{Hom}_{\mathscr{H}}\left(M\left(v^{t}\right), M\left(\mu^{t}\right)\right)$. Then $\theta^{t}\left(\varphi_{U^{t} T^{v^{t}}}\right)=0$ by assumption and hence

$$
\sum_{A^{t}} \sum_{a^{t}} \gamma_{A^{t}} m_{\lambda^{t}} T_{d\left(a^{t}\right)} h^{t}=\left[\theta^{t}\left(\varphi_{A^{t} T^{v^{t}}}\right)\right]\left(m_{v^{t}}\right) \in \check{\mathscr{H}}^{\lambda^{t}}
$$

Also,

$$
\sum_{A^{t}} \sum_{a^{b}} \gamma_{A^{t}} m_{\lambda^{b}} T_{d\left(a^{b}\right)} h^{b} \in M\left(\lambda^{b}\right) .
$$

Hence we can write

$$
\begin{aligned}
{\left[\theta\left(\varphi_{U V}\right)\right]\left(m_{\tau}\right)=} & \check{\mathscr{H}}^{\lambda}+\left(\sum_{x^{t}, y^{t}}\left\{\delta_{x^{t} y^{t}} m_{x^{t} y^{t}} \mid x^{t}, y^{t} \in \operatorname{Std}\left(\tau^{t}\right) \text { where } \tau^{t} \triangleright \lambda^{t}\right\}\right) \\
& \times\left(\sum_{x^{b}, y^{b}}\left\{\delta_{x^{b} y^{b}} m_{x^{b} y^{b}} \mid x^{b}, y^{b} \in \operatorname{Std}\left(\tau^{b}\right) \text { where } \tau^{b} \unrhd \lambda^{b}\right\}\right) T_{d(t)}
\end{aligned}
$$


for some $\delta_{x^{t} y^{t}}, \delta_{x^{b} y^{b}} \in R$. Now for any such $m_{x^{t} y^{t}}, m_{x^{b} y^{b}}$ we may write

$$
\begin{aligned}
m_{x^{t} y^{t}} m_{x^{b} y^{b}} & =T_{d\left(x^{t}\right)}^{*} m_{\tau^{t}} T_{d\left(y^{t}\right)} T_{d\left(x^{b}\right)}^{*} m_{\tau^{b}} T_{d\left(y^{b}\right)} \\
& =T_{d\left(x^{t}\right)}^{*} T_{d\left(x^{b}\right)}^{*} m_{\tau^{t}} m_{\tau^{b}} T_{d\left(y^{t}\right)} T_{d\left(y^{b}\right)} \\
& =T_{d\left(x^{t}, x^{b}\right)}^{*} m_{\tau} T_{d\left(y^{t}, y^{b}\right)}
\end{aligned}
$$

where $\tau=\left(\tau_{1}^{t}, \tau_{2}^{t}, \ldots, \tau_{1}^{b}, \tau_{2}^{b}, \ldots\right)$ is a composition of $n$ such that $\tau \triangleright \lambda$ and $\left(x^{t}, x^{b}\right)$, $\left(y^{t}, y^{b}\right)$ are row standard $\tau$-tableaux. By Definition 2.1, this can be written as an $R$-linear combination of terms $m_{u v}$ where $u, v \in \operatorname{Std}\left(\tau^{\prime}\right)$ and $\tau^{\prime} \unrhd \tau \triangleright \lambda$. Hence $m_{x^{t} y^{\prime}} m_{x^{b} y^{b}} \in \breve{\mathscr{H}}^{\lambda}$. Since $\breve{\mathscr{C}}^{\lambda}$ is a right ideal, it follows that $m_{x^{t} y^{t}} m_{x^{b}{ }^{b}{ }^{b}} T_{d(t)} \in \breve{\mathscr{H}}^{\lambda}$ and so $\left[\theta\left(\varphi_{U V}\right)\right]\left(m_{\tau}\right)=0$ as required.

Proposition 3.14 Suppose that $\theta^{t} \in \operatorname{Hom}_{\mathcal{S}^{t}}\left(\mathcal{M}\left(\mu^{t}\right), \Delta\left(\lambda^{t}\right)\right)$, and $\theta^{b} \in \operatorname{Hom}_{\mathcal{S}^{b}}\left(\mathcal{M}\left(\mu^{b}\right)\right.$, $\left.\Delta\left(\lambda^{b}\right)\right)$ are such that

$$
\theta^{t}\left(\varphi_{\mu^{t}} f\right)=0 \quad \text { whenever } f \in \mathcal{S}^{t} \text { and } \varphi_{\mu^{t}} f \in \check{\mathcal{S}}^{\mu^{t}}
$$

and

$$
\theta^{b}\left(\varphi_{\mu^{b}} f\right)=0 \quad \text { whenever } f \in \mathcal{S}^{b} \text { and } \varphi_{\mu^{b}} f \in \check{\mathcal{S}}^{\mu^{b}} .
$$

Define the map $\theta=\left(\theta^{t}, \theta^{b}\right)$ as in Definition 3.9, and define the map $\hat{\theta}: \Delta(\mu) \rightarrow \Delta(\lambda)$ by

$$
\hat{\theta}\left[\left(\check{\mathcal{S}}^{\mu}+\varphi_{\mu}\right) f\right]=\left[\theta\left(\varphi_{\mu}\right)\right] f \quad \text { for all } f \in \mathcal{S} .
$$

Then $\hat{\theta}$ is a well defined $\mathcal{S}$-homomorphism.

Proof: It is sufficient to show that if $f \in \mathcal{S}$ is such that $\varphi_{\mu} f \in \check{\mathcal{S}}^{\mu}$ then $\left[\theta\left(\varphi_{\mu}\right)\right] f=$ $\theta\left(\varphi_{\mu} f\right)=0$. Suppose $f$ satisfies these conditions. Then $\varphi_{\mu} f$ is an $R$-linear combination of terms of the form $\varphi_{U V}$ where $U \in \mathcal{T}_{0}(\nu, \mu)$ and $V \in \mathcal{T}_{0}(\nu, \tau)$ for some $\nu \in \Lambda^{+}(d, n), \tau \in$ $\Lambda(d, n)$ such that $v \triangleright \mu$. Choose such an $\varphi_{U V}$. If $\lambda \unrhd v$ then $\theta\left(\varphi_{U V}\right)=0$ by Proposition 3.12. If $\lambda \unrhd v$ then $\theta\left(\varphi_{U V}\right)=0$ by Proposition 3.13. Hence $\theta\left(\varphi_{\mu} f\right)=0$ as required.

Definition 3.15 Suppose that

$$
\hat{\theta}^{t} \in \operatorname{Hom}_{\mathcal{S}^{t}}\left(\Delta\left(\mu^{t}\right), \Delta\left(\lambda^{t}\right)\right), \quad \text { and } \quad \hat{\theta}^{b} \in \operatorname{Hom}_{\mathcal{S}^{b}}\left(\Delta\left(\mu^{b}\right), \Delta\left(\lambda^{b}\right)\right) .
$$

Define

$$
\theta^{t} \in \operatorname{Hom}_{\mathcal{S}^{t}}\left(\mathcal{M}\left(\mu^{t}\right), \Delta\left(\lambda^{t}\right)\right), \quad \text { and } \quad \theta^{b} \in \operatorname{Hom}_{\mathcal{S}^{b}}\left(\mathcal{M}\left(\mu^{b}\right), \Delta\left(\lambda^{b}\right)\right) .
$$

in the obvious manner; that is, $\theta^{t}=\hat{\theta}^{t} \circ \pi^{t}$ and $\theta^{b}=\hat{\theta}^{b} \circ \pi^{b}$ where $\pi_{T}$ and $\pi_{B}$ are the projections onto $\Delta\left(\mu^{t}\right)$ and $\Delta\left(\mu^{b}\right)$ respectively. Note that $\theta^{t}$ and $\theta^{b}$ satisfy the conditions of Proposition 3.14. Hence define

$$
\hat{\theta}=\left(\hat{\theta}^{t}, \hat{\theta}^{b}\right) \in \operatorname{Hom}_{\mathcal{S}}(\Delta(\mu), \Delta(\lambda))
$$

as in Proposition 3.14. 
Proposition 3.16 Let $\theta \in \operatorname{Hom}_{\mathcal{S}}(\mathcal{M}(\mu), \Delta(\lambda))$ be such that

$$
\theta\left(\varphi_{\mu} f\right)=0 \quad \text { whenever } f \in \mathcal{S} \quad \text { and } \quad \varphi_{\mu} f \in \check{\mathcal{S}}^{\mu}
$$

Then by Lemma 3.7 there exist $\gamma_{A^{t} A^{b}} \in R$ such that

$$
\theta=\sum_{A^{t}, A^{b}}\left\{\gamma_{A^{t} A^{b}} \theta_{\left(A^{t}, A^{b}\right)} \mid A^{t} \in \mathcal{T}_{0}\left(\lambda^{t}, \mu^{t}\right), A^{b} \in \mathcal{T}_{0}\left(\lambda^{b}, \mu^{b}\right)\right\}
$$

Fix $A^{b}$ and consider $\theta^{t}=\sum_{A^{t}} \gamma_{A^{t} A^{b}} \theta_{A^{t}} \in \operatorname{Hom}_{\mathcal{S}^{t}}\left(\mathcal{M}\left(\mu^{t}\right), \Delta\left(\lambda^{t}\right)\right)$. Then

$$
\theta^{t}\left(\varphi_{\mu^{t}} f\right)=0 \quad \text { whenever } f \in \mathcal{S}^{t} \quad \text { and } \quad \varphi_{\mu^{t}} f \in \check{\mathcal{S}}^{\mu^{t}}
$$

Proof: Suppose we have the conditions of Proposition 3.16. By Proposition 3.12 and Proposition 3.14, it is sufficient to show that $\theta^{t}\left(\varphi_{U V}\right)=0$ for any $U \in \mathcal{T}_{0}\left(v^{t}, \mu^{t}\right), V=T^{v^{t}}$ such that $v^{t} \in \Lambda^{+}(d, n)$ and $\lambda^{t} \unrhd v^{t} \triangleright \mu^{t}$. We have

$$
\sum_{u}\left\{T_{d(u)}^{*} m_{v^{t}} \mid u \in \operatorname{Std}\left(v^{t}\right) \text { and } \mu^{t}(u)=U\right\}=\mathrm{m}_{\mu^{t}} h^{t}
$$

for some $h^{t} \in \mathscr{H}^{t}$. Then $\theta^{t}\left(\varphi_{U V}\right)=0$ if and only if

$$
\sum_{A^{t}} \gamma_{A^{t} A^{b}} \sum_{a^{t}}\left\{m_{\lambda^{t}} T_{d\left(a^{t}\right)} h^{t} \mid a^{t} \in \operatorname{Std}\left(\lambda^{t}\right) \text { and } \mu^{t}\left(a^{t}\right)=A^{t}\right\} \in \check{\mathscr{H}}^{\lambda^{t}}
$$

Now fix $v=\left(v^{t}, v^{b}\right), X=\left(U^{t}, T^{v^{b}}\right), Y=T^{v}$. By assumption, $\theta\left(\varphi_{X Y}\right)=0$. Hence

$$
\sum_{A^{t}, A^{b}} \gamma_{A^{t} A^{b}} \sum_{a^{t}} \sum_{a^{b}}\left\{m_{\lambda^{t}} m_{\lambda^{b}} T_{d\left(a^{t}\right)} T_{d\left(a^{b}\right)} h^{t} \mid a^{b} \in \operatorname{Std}\left(\lambda^{b}\right) \text { and } \mu^{b}\left(a^{b}\right)=A^{b}\right\} \in \check{\mathscr{H}}^{\lambda}
$$

and so

$$
\sum_{A^{t}} \gamma_{A^{t} A^{b}} \sum_{a^{t}} m_{\lambda^{t}} T_{d\left(a^{t}\right)} h^{t} \in \check{\mathscr{H}}^{\lambda^{t}}
$$

as required.

Definition 3.17 Suppose $\theta \in \operatorname{Hom}_{\mathscr{H}}(\mathcal{M}(\mu), \Delta(\lambda))$ is such that $\theta\left(\varphi_{\mu} f\right)=0$ for all $f \in \mathcal{S}$ such that $\varphi_{\mu} f \in \check{\mathcal{S}}^{\mu}$. Define $\hat{\theta} \in \operatorname{Hom}_{\mathcal{S}}(\Delta(\mu), \Delta(\lambda))$ by setting

$$
\hat{\theta}\left(\varphi_{T^{\mu}} f\right)=\theta\left(\varphi_{\mu}\right) f, \quad \text { for all } f \in \mathcal{S} \text {. }
$$


Similarly, if $\hat{\theta} \in \operatorname{Hom}_{\mathcal{S}}(\Delta(\mu), \Delta(\lambda))$, define $\theta \in \operatorname{Hom}_{\mathcal{S}}(\mathcal{M}(\mu), \Delta(\lambda))$ by $\theta=\hat{\theta} \circ \pi$ where $\pi: \mathcal{M}(\mu) \rightarrow \Delta(\mu)$ is the natural projection. We use analogous notation for the corresponding $\mathcal{S}^{t}$ and $\mathcal{S}^{b}$ homomorphisms.

We can now prove Theorem 3.1.

Proof of Theorem 3.1: We have to show that

$$
\operatorname{Hom}_{\mathcal{S}}(\Delta(\mu), \Delta(\lambda)) \cong \operatorname{Hom}_{\mathcal{S}^{t}}\left(\Delta\left(\mu^{t}\right), \Delta\left(\lambda^{t}\right)\right) \otimes \operatorname{Hom}_{\mathcal{S}^{b}}\left(\Delta\left(\mu^{b}\right), \Delta\left(\lambda^{b}\right)\right) .
$$

Let $\hat{\theta}^{t} \in \operatorname{Hom}_{\mathscr{H}^{c}}\left(\Delta\left(\mu^{t}\right), \Delta\left(\lambda^{t}\right)\right)$ and $\hat{\theta}^{b} \in \operatorname{Hom}_{\mathcal{S}^{b}}\left(\Delta\left(\mu^{b}\right), \Delta\left(\lambda^{b}\right)\right)$. Then there is an $R$-linear mapping from $\operatorname{Hom}_{\mathcal{S}^{t}}\left(\Delta\left(\mu^{t}\right), \Delta\left(\lambda^{t}\right)\right) \otimes \operatorname{Hom}_{\mathcal{S}^{b}}\left(\Delta\left(\mu^{b}\right), \Delta\left(\lambda^{b}\right)\right)$ into $\operatorname{Hom}_{\mathcal{S}}(\Delta(\mu), \Delta(\lambda))$ given by sending

$$
\hat{\theta}^{t} \otimes \hat{\theta}^{b} \mapsto\left(\hat{\theta}^{t}, \hat{\theta}^{b}\right)
$$

and extending linearly. By construction, this mapping is injective. It remains only to show that it is surjective.

Let $\hat{\theta} \in \operatorname{Hom}_{\mathcal{S}}(\Delta(\mu), \Delta(\lambda))$. Form $\theta=\hat{\theta} \circ \pi \in \operatorname{Hom}_{\mathcal{S}}(\mathcal{M}(\mu), \Delta(\lambda))$ so that $\theta$ satisfies the conditions of Proposition 3.16. Suppose

$$
\theta=\sum_{A^{t}, A^{b}}\left\{\gamma_{A^{t} A^{b}} \theta_{\left(A^{t}, A^{b}\right)} \mid A^{t} \in \mathcal{T}_{0}\left(\lambda^{t}, \mu^{t}\right), A^{b} \in \mathcal{T}_{0}\left(\lambda^{b}, \mu^{b}\right)\right\}
$$

for some $\gamma_{A^{t} A^{b}} \in R$. Fix $A^{b}$. By Proposition 3.16, the map

$$
\theta^{t}=\sum_{A^{t}} \gamma_{A^{t} A^{b}} \theta_{A^{t}}
$$

is such that the corresponding map $\hat{\theta}^{t}: \Delta\left(\mu^{t}\right) \rightarrow \Delta\left(\lambda^{t}\right)$ is well defined. Suppose that $\left\{\hat{\psi}_{i} \mid i \in I\right\}$ is a basis of $\operatorname{Hom}_{\mathcal{S}^{t}}\left(\Delta\left(\mu^{t}\right), \Delta\left(\lambda^{t}\right)\right)$. So we can write

$$
\hat{\theta}^{t}=\sum_{i} \beta_{i, A^{b}} \hat{\psi}_{i}
$$

and so

$$
\theta=\sum_{i, A^{b}} \beta_{i, A^{b}}\left(\psi_{i}, \theta_{A^{b}}\right)
$$

A similar argument for fixed $\psi_{i}$ shows that in fact

$$
\theta=\sum_{i, j} \alpha_{i j}\left(\psi_{i}^{t}, \psi_{j}^{b}\right)
$$


where $\hat{\psi}_{i}^{t} \in \operatorname{Hom}_{\mathcal{S}^{t}}\left(\Delta\left(\mu^{t}\right), \Delta\left(\lambda^{t}\right)\right)$ and $\hat{\psi}_{j}^{b} \in \operatorname{Hom}_{\mathcal{S}^{b}}\left(\Delta\left(\mu^{b}\right), \Delta\left(\lambda^{b}\right)\right)$; thus under our mapping, $\hat{\theta}$ appears as the image of

$$
\sum_{i, j} \alpha_{i j}\left(\hat{\psi}_{i}^{t} \otimes \hat{\psi}_{j}^{b}\right) \in \operatorname{Hom}_{\mathcal{S}^{t}}\left(\Delta\left(\lambda^{t}\right), \Delta\left(\mu^{t}\right)\right) \otimes \operatorname{Hom}_{\mathcal{S}^{b}}\left(\Delta\left(\lambda^{b}\right), \Delta\left(\mu^{b}\right)\right) .
$$

\section{The proof of Theorem 1.5}

In this section we give Donkin's proof of Theorem 1.5. In fact the argument is a generalization of Donkin's proof of Corollary 1.6 in the classical case; see [15, Section 4]. See also [13, Section 4.2(16) and (17)] and compare with [5, Remark 11(b)].

Throughout this section we assume that $R$ is a field and we use the notation and terminology from Donkin's book [13], especially from section 4.2. Although we have tried to make our exposition self-contained, we refer the reader to [13] for further explanation of the notation and concepts used below.

Recall from the introduction that $G(d)$ is the quantum general linear group, $\Pi$ is a set of simple roots for $G(d)$ and $G_{\Sigma}(d)$ is the Levi quantum subgroup corresponding to $\Sigma \subset \Pi$. Let $P_{\Sigma}(d)$ be the corresponding parabolic quantum subgroup of $G(d)$. Let $X$ be the set of weights for $G(d)$ and $X^{+}$the set of dominant weights, and let $X_{\Sigma}$ and $X_{\Sigma}^{+}$be the corresponding weights for $G_{\Sigma}(d)$. Recall that for each $\lambda \in X^{+}$we have the exact functor

$$
\operatorname{Tr}_{\Sigma}^{\lambda}: \operatorname{Mod}-G(d) \rightarrow \operatorname{Mod}-G_{\Sigma}(d) ; V \mapsto \bigoplus_{\substack{\mu \in X \\ \lambda-\mu \in \mathbb{Z} \Sigma}} V_{\mu}
$$

Observe that we can regard any $G_{\Sigma}(d)$-module as a $P_{\Sigma}(d)$-module via inflation using the natural homomorphism $P_{\Sigma}(d) \rightarrow G_{\Sigma}(d)$; in particular, we can think of $\operatorname{Tr}_{\Sigma}^{\lambda} V$ as a $P_{\Sigma}(d)$ module.

Now fix $\lambda \in X^{+}$and suppose that $V=\oplus V_{\tau}$ is a $G(d)$-module such that $V_{\tau}$ is non-zero only if $\lambda \geq \tau$. Set $V_{0}=\oplus_{\lambda-\tau \notin \mathbb{Z} \Sigma} V_{\tau}$. Then $V_{0}$ is a $P_{\Sigma}(d)$ submodule of $V$ and it is easy to see that $\operatorname{Tr}_{\Sigma}^{\lambda} V \cong V / V_{0}$ as $P_{\Sigma}(d)$-modules.

Rather than prove the version of Theorem 1.5 stated in the introduction we prove the equivalent statement for the induced modules $\nabla(\mu) \cong \Delta\left(-w_{0} \mu\right)^{*}$, where $\mu \in X^{+}$and $w_{0}$ is the element of longest length in the Weyl group $\mathfrak{S}_{n}$.

Let $G$ be one of the quantum groups (Hopf algebras) $G(d), G_{\Sigma}(d)$ or $P_{\Sigma}(d)$. Recall that if $W$ and $V$ are finite dimensional $G$-modules then $\operatorname{Ext}_{G}^{k}(V, W)=H^{k}\left(G, V^{*} \otimes W\right)$, where $H^{k}(G, \ldots)$ is the $k$ th right derived functor of the fixed point functor from Mod- $G$ to $R$-vector spaces.

We will also need the following result. Suppose that $V$ and $W$ are finite dimensional $G_{\Sigma}(d)$-modules such $\tau-\sigma \in \mathbb{Z} \Sigma$ whenever $V_{\tau} \neq 0$ and $W_{\sigma} \neq 0$. Then, by [13, Section 4.2(16)],

$$
\operatorname{Ext}_{G_{\Sigma}(d)}^{k}(V, W)=H^{k}\left(G_{\Sigma}(d), V^{*} \otimes W\right) \cong H^{k}\left(P_{\Sigma}(d), V^{*} \otimes W\right)=\operatorname{Ext}_{P_{\Sigma}(d)}^{k}(V, W) .
$$


We can now give the proof of Donkin's result.

Theorem 4.2 (Donkin) Let $R$ be a field, $\Sigma \subset \Pi$ and let $\lambda$ and $\mu$ be dominant weights such that $\lambda-\mu \in \mathbb{Z} \Sigma$. Suppose that $V=\oplus V_{\tau}$ is a finite dimensional $G(d)$-module such that $V_{\tau} \neq 0$ only if $\lambda \geq \tau$. Then

$$
\operatorname{Ext}_{G(d)}^{k}(V, \nabla(\mu)) \cong \operatorname{Ext}_{G_{\Sigma}(d)}^{k}\left(\operatorname{Tr}_{\Sigma}^{\lambda} V, \nabla_{\Sigma}(\mu)\right)
$$

for all $k \geq 0$.

Proof: If $\operatorname{Ext}_{G_{\Sigma}(d)}^{k}\left(\operatorname{Tr}_{\Sigma}^{\lambda} V, \nabla_{\Sigma}(\mu)\right) \neq 0$ then $\operatorname{Ext}_{G_{\Sigma}(d)}^{k}\left(L_{\Sigma}(\tau), \nabla_{\Sigma}(\mu)\right) \neq 0$ for some composition factor $L_{\Sigma}(\tau)$ of $\operatorname{Tr}_{\Sigma}^{\lambda} V$. Therefore, $\tau \geq \mu$ and, as $\lambda \geq \tau$, we also have $\lambda \geq \mu$. Applying this to the case $\Sigma=\Pi$ we find, in particular, that $\lambda \geq \mu$ if $\operatorname{Ext}_{G(d)}^{k}(V, \nabla(\mu)) \neq 0$. Hence we may assume, in proving the Theorem, that $\lambda \geq \mu$.

By definition, if $\tau$ is a weight of $\operatorname{Tr}_{\Sigma}^{\lambda} V$ then $\lambda-\tau \in \mathbb{Z} \Sigma$. On the other hand, if $\sigma$ is a weight of $\nabla_{\Sigma}(\mu)$ then $\mu-\sigma \in \mathbb{Z} \Sigma$ by the remarks after [13, Section 4.2(1)]. As $\lambda-\mu \in$ $\mathbb{Z} \Sigma$ it follows that $\operatorname{Tr}_{\Sigma}^{\lambda} V$ and $W=\nabla_{\Sigma}(\mu)$ satisfy the hypothesis of (4.1); consequently, $\operatorname{Ext}_{G_{\Sigma}(d)}^{k}\left(\operatorname{Tr}_{\Sigma}^{\lambda} V, \nabla_{\Sigma}(\mu)\right) \cong \operatorname{Ext}_{P_{\Sigma}(d)}^{k}\left(\operatorname{Tr}_{\Sigma}^{\lambda} V, \nabla_{\Sigma}(\mu)\right)$. On the other hand, $\operatorname{Ext}_{G(d)}^{k}(V, \nabla(\mu)) \cong$ $\operatorname{Ext}_{P_{\Sigma}(d)}^{k}\left(V, \nabla_{\Sigma}(\mu)\right)$ by a corollary of Kempf's vanishing theorem (see [26, Section II.4.6(a)] or [10, 2.1.3]). So it remains to prove that $\operatorname{Ext}_{P_{\Sigma}(d)}^{k}\left(V, \nabla_{\Sigma}(\mu)\right) \cong \operatorname{Ext}_{P_{\Sigma}(d)}^{k}\left(\operatorname{Tr}_{\Sigma}^{\lambda} V, \nabla_{\Sigma}(\mu)\right)$.

As remarked above, $\operatorname{Tr}_{\Sigma}^{\lambda} V \cong V / V_{0}$ as a $P_{\Sigma}(d)$-module, so we have a short exact sequence of $P_{\Sigma}(d)$-modules $0 \rightarrow V_{0} \rightarrow V \rightarrow \operatorname{Tr}_{\Sigma}^{\lambda} V \rightarrow 0$. Therefore, by the induced long exact sequence, it suffices to prove that $\operatorname{Ext}_{P_{\Sigma}(d)}^{k}\left(V_{0}, \nabla_{\Sigma}(\mu)\right)=0$, for $k \geq 0$. Thus, by another long exact sequence argument, we are reduced to showing that $\operatorname{Ext}_{P_{\Sigma}(d)}^{k}\left(L_{\Sigma}(\tau), \nabla_{\Sigma}(\mu)\right)=0$ for each $P_{\Sigma}(d)$ composition factor $L_{\Sigma}(\tau)$ of $V_{0}$. Suppose, if possible, that $\operatorname{Ext}_{P_{\Sigma}(d)}^{k}\left(L_{\Sigma}(\tau)\right.$, $\left.\nabla_{\Sigma}(\mu)\right) \neq 0$. Then $\tau \geq \mu$, so $\tau-\mu$ is a sum of positive roots. We trivially have $\lambda-\mu=$ $(\lambda-\tau)+(\tau-\mu)$; however, notice that both $\lambda-\tau$ and $\tau-\mu$ are sums of positive roots, so no cancellation can occur in this sum. Consequently, we have $\lambda-\tau \in \mathbb{Z} \Sigma$ since $\lambda-\mu \in \mathbb{Z} \Sigma$. However, this contradicts the definition of $V_{0}$ and so completes the proof.

Remark The proof of Theorem 4.2 relies, ultimately, on Kempf's vanishing theorem. The argument is equally valid for any reductive algebraic group $G$ (compare [5, Corollary 10]), and for any quantized enveloping algebra for which a version of Kempf's vanishing theorem holds.

\section{Acknowledgments}

We are grateful to Stephen Donkin for many useful comments and for allowing us to include his proof of Theorem 4.2. We also thank the referees for drawing the papers $[5,14]$ to our attention, Leonard Scott for sending us a copy of [4], and Marco Soriano and Alison Parker for their comments on an earlier version of this paper. 


\section{References}

1. J. Brundan, R. Dipper, and A.S. Kleshchev, "Quantum linear groups and representations of $G L_{n}\left(\mathbb{F}_{q}\right)$," Memoirs A.M.S. 706, (2001).

2. R.W. Carter and G. Lusztig, "On the modular representations of the general linear and symmetric groups," Math. Z. 136 (1974), 193-142.

3. J. Chuang, H. Miyachi, and K.M. Tan, "Row and column removal in the $q$-deformed Fock space," J. Algebra 254 (2002), 84-91.

4. E. Cline, B. Parshall, and L. Scott, "Abstract Kazhdan-Lusztig theories," Tôhoku Math. J. 45 (1993), $511-$ 534.

5. E. Cline, B. Parshall, and L. Scott, "On Ext-transfer for algebraic groups," Trans. Groups 9 (2004), 213236.

6. R. Dipper and G. James, "Representations of Hecke algebras of general linear groups," Proc. L.M.S. (3) 52 (1986), 20-52.

7. R. Dipper and G. James, "The $q$-Schur algebra," Proc. L.M.S. (3) 59 (1989), 23-50.

8. R. Dipper and G. James, " $q$-Tensor space and $q$-Weyl modules," Trans. A.M.S. 327 (1991), 251-282.

9. S. Donkin, "A note of decomposition numbers for general linear groups and symmetric groups," Math. Proc. Cambridge Phil. Soc. 97 (1985), 57-62.

10. S. Donkin, "Rational Representations of Algebraic Groups: Tensor Products and Filtrations, SLN 1140, Springer-Verlag, 1985.

11. S. Donkin, "On Schur algebras and related algebras I," J. Algebra 104 (1986), 310-328.

12. S. Donkin, "Standard homological properties for quantum $G L_{n}$," J. Algebra 181 (1996), 235-266.

13. S. Donkin, The $q$-Schur algebra, L.M.S. Lecture Notes 253, CUP, Cambridge, 1999.

14. S. Donkin, "Tilting modules for algebraic groups and finite dimensional algebras," in The handbook of Tilting Theory, D. Happel and H. Krause (Eds.), Cambridge University Press, to appear, 2004.

15. K. Erdmann, "Ext ${ }^{1}$ for Weyl modules of $S L_{2}(K)$," Math. Z. 218 (1995), 447-459.

16. M. Fayers and S. Lyle, "Row and column removal theorems for homomorphisms between Specht modules," J. Pure Appl. Algebra 185 (2003), 147-164.

17. J.J. Graham and G.I. Lehrer, "Cellular algebras," Invent. Math. 123 (1996), 1-34.

18. J.A. Green, Polynomial Representations of $G L_{n}$, SLN vol. 830, Springer-Verlag, New York, 1980.

19. D.J. Hemmer, "Fixed-point functors for symmetric groups and Schur algebras," J. Algebra $\mathbf{2 8 0}$ (2004), 295312.

20. D.J. Hemmer, "A row removal theorem for the ext ${ }^{1}$ quiver of symmetric groups and Schur algebras," $J$. Algebra 280 (2004), 295-312.

21. D.J. Hemmer and D.K. Nakano, "Specht filtrations for Hecke algebras of type A," J. London Math. Soc. (2) 69 (2004), 623-638

22. G.D. James, The Representation Theory of the Symmetric Groups, SLN 682, Springer-Verlag, New York, 1978.

23. G.D. James, "On the decomposition matrices of the symmetric groups III," Math. Z. 71 (1981), 115-122.

24. G.D. James, "Representations of General Linear Groups," L.M.S. Lecture Notes 94, CUP, 1984.

25. G.D. James, "The decomposition matrices of $G L_{n}(q)$ for $n \leq 10$," Proc. L.M.S. (3) 60 (1990), 225-264.

26. J.C. Jantzen, Representations of Algebraic Groups, Mathematical Surveys and Monographs 107, American Mathematical Society, Providence, RI, second ed., 2003.

27. M. Kashiwara and T. Tanisaki, "Kazhdan-lusztig conjecture for affine algebras with negative level," Duke J. Math. 77 (1995), 21-62.

28. D. Kazhdan and G. Lusztig, "Tensor structures arising from affine lie algebras I-IV," J. A.M.S. 6 (1993), 9-1011 (7) (1994), 335-453.

29. A.S. Kleshchev and J. Sheth, "On extensions of simple modules over symmetric and algebraic groups," $J$. Algebra 221 (1999), 705-722.

30. A. Lascoux, B. Leclerc, and J.-Y. Thibon, "Hecke algebras at roots of unity and crystal bases of quantum affine algebras," Comm. Math. Phys. 181 (1996), 205-263.

31. B. Leclerc, "Decomposition numbers and canonical bases," Alg. Represent. Theory 3 (2000), 277287. 
32. B. Leclerc and J.-Y. Thibon, "Littlewood-Richardson coefficients and Kazhdan-Lusztig polynomials," in Combinatorial methods in representation theory, M. Kashiwara et al., Eds., Adv. Pure Math. vol. 28, 2000, pp. $155-220$

33. A. Mathas, "Hecke algebras and Schur algebras of the symmetric group," Univ. Lecture Notes 15, A.M.S., (1999).

34. G.E. Murphy, "The representations of Hecke algebras of type A $\mathbf{A}_{\mathbf{n}}$, J. Algebra 173 (1995), 97-121.

35. M. Varagnolo and E. Vasserot, "On the decomposition matrices of the quantized Schur algebra," Duke J. Math. 100 (1999), 267-297. 